\title{
Research Paper \\ Comparing the Effect of Kinesiology Taping on Dynamic Balance and Pain of Men and Women With Unilateral Patellofemoral Pain Syndrome
}

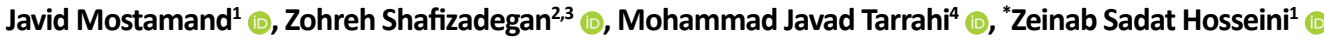

1. Department of Physical Therapy, Musculoskeletal Research Center, Faculty of Rehabilitation Sciences, Isfahan University of Medical Sciences, Isfahan, Iran

2. Department of Physical Therapy, School of Rehabilitation Sciences, Iran University of Medical Sciences, Tehran, Iran.

3. Department of Physical Therapy, School of Rehabilitation Sciences, Isfahan University of Medical Sciences, Isfahan, Iran

4. Department of Epidemiology and Statistics, School of Health, Isfahan University of Medical Science, Isfahan, Iran.

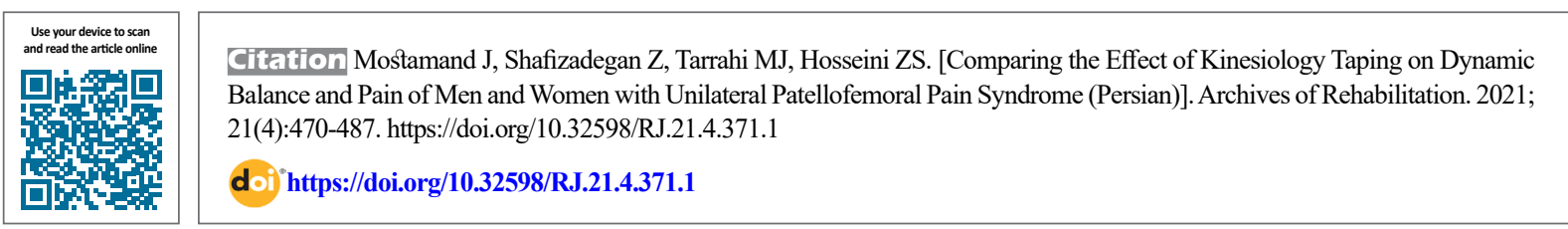

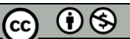

Received: 28 Jul 2019

Accepted: 09 Aug 2020

Available Online: 01 Jan 2021
Keywords:

Patellofemoral pain syndrome, Kinesio tape, Postural balance, Patellofemoral syndrome

\section{A BSTRACT}

Objective Patellofemoral Pain Syndrome (PFPS) is one of the most common disorders of the knee joint. It is characterized by pain, reduced proprioception, and altered pattern of vastus muscle activation, which effectively maintain the balance needed for performing daily living activities such as walking and running. One treatment method that can reduce pain and improve balance in people with PFPS is Kinesiology Taping (KT). Considering the physiological and anatomical differences in the knee structure of men and women and the importance of studying the effect of KT on the dynamic balance of men with PFPS, this study aims to compare the effect of KT on dynamic balance and pain of men and women with unilateral PFPS. Materials \& Methods This is a quasi-experimental and non-randomized clinical trial. The participants were 30 males and 31 females aged $18-40$ years suffering from unilateral PFPS. They were recruited using a convenience sampling method. First, the subjects performed the Y-balance test three times in each of the anterior, posteromedial, and posterolateral directions. The maximum score obtained from the three repetitions in each direction was divided by the limb length and recorded in percentage as a dynamic balance score. After 5 minutes of rest and performing initial tests, the subjects performed the intervention. In this stage, KT with a tension equal to $50 \%-75 \%$ of its initial length was applied on the patella of the involved limb to cause medial glide. The effectiveness of $\mathrm{KT}$ was examined under a single-leg squat-test. For this purpose, all subjects performed unilateral squatting on their affected leg for 10 seconds with 45 degrees of knee flexion before and after $\mathrm{KT}$, while their pain level was recorded using the visual analog scale. In case of a $50 \%$ reduction in pain, the subjects were allowed to enter the final stage (performing the $\mathrm{Y}$-balance test); otherwise, patellar taping was repeated to obtain the appropriate pain reduction.

Results Within-group comparison of balance parameters before and after KT using the paired t-test showed an increase in the reach distance at three directions in the $Y$-balance test, revealing a significant improvement in the dynamic balance following KT $(P<0.05)$. According to the independent $t$-test results, there was no significant difference in balance parameters between males and females before and after the intervention ( $P>0.05)$. Moreover, the pain was significantly reduced in both genders after KT $(P<0.05)$, but its difference between men and women was not statistically significant $(P>0.05)$.

Conclusion KT is an appropriate therapeutic intervention for improving dynamic balance and reducing pain in people with PFPS. It seems that KT has the same effect on dynamic balance and pain of women and men with PFPS.

"Corresponding Author:

Zeinab Sadat Hosseini

Address: Department of Physical Therapy, School of Rehabilitation Sciences, Iran University of Medical Sciences, Tehran, Iran.

Tel: +98 (913) 3186217

E-Mail: zainab.hosseini62@gmail.com 


\section{Extended Abstract}

\section{Introduction}

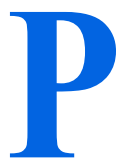

ain in the anterior and posterior part of the patella, without the presence of other pathologies of the knee joint, is called Patellofemoral Pain Syndrome (PFPS) [1, 2].

One of the consequences of this syndrome is a change in balance control [4-8]. One of the influential anatomical factors in the patellar joint's stability is the balance between the internal and external vas deferens muscles. After decreasing quadriceps muscle activity and disrupting the contraction sequence of these muscles in PFPS, joint stability indicators also change. Therefore, the afferents of muscle receptors alter, and knee joint proprioception, the most crucial sensory source for balance, is damaged [9-12]. Although muscular inhibition does not directly impair proprioception, it can impair neuromuscular control mechanisms in people with PFPS [13]. Regulating the proprioception in people with this PFPS helps to increase the patient's performance and accelerate the rehabilitation process [14]. The results of previous studies have indicated a change in proprioception and a decrease in dynamic balance in these patients [15-18]. Hence, it is necessary to pay attention to improving proprioception and dynamic balance in rehabilitating people with PFPS.

One of the treatment techniques in physiotherapy that improves balance in people with PFPS is the use of kinesio taping. It can treat imbalance between the internal and external vas deferens muscles $[19,20]$. It also improves the decreased proprioception in people with PFPS [21]. Since women's knee joints are looser than men's, it is expected that women's knee joint proprioception decrease and their risk of injury increase, which can make a significant difference in balance between men and women [22, 23]. Neuromuscular control during physical movements has also been reported differently in male and female adolescents [24]. Besides, the presence of some hormonal, anatomical, and neuromuscular factors in women has made PFPS more common in women [12, 22, 25]. To our knowledge, there is no study, neither on the effect of kinesio taping on dynamic balance in men with PFPS nor on comparing its effect on dynamic balance and pain in non-athlete men and women with this syndrome. Therefore, considering the mentioned physiological and anatomical differences between men's and women's knees, it seems necessary to study the effect of kinesio taping on dynamic balance in men with PFPS and compare it with women. Given the importance of balance in daily activities, this study aimed to compare the effect of kinesio taping on pain and dynamic balance between non-athlete men and women with unilateral PFPS.

\section{Materials and Methods}

This study was performed with the approval of the Ethics Committee of Isfahan University of Medical Sciences and in the physiotherapy clinic of Shahid Sadoughi Hospital in Isfahan City, Iran. The participants were 30 men and 31 women aged 18-40 years with unilateral PFPS who were selected using a convenience sampling method. The inclusion criteria were the existence of pain in the back of the patella or anterior knee, exacerbated during at least two activities of walking, running, jumping, stair climbing, and prolonged kneeling and sitting [26]; unilateral PFPS [27]; a pain score more than 3 under single-leg squat-test on the involved leg up to a 45-degree angle for 10 seconds [28]; and the existence of external glide based on McConnell test [29]. The exclusion criteria were receiving any type of rehabilitation for the knee joint in the past three months [30]; participating in a particular sport; gradual onset of pain for at least 8 weeks [27]; surgery on the knee, lower back, hip, or lower limbs [31-33], traumatic, inflammatory, infectious disorders, fracture or deformation in the knee and lower limbs [27, 28]; patellar dislocation and subluxation and any symptoms related to the presence of knee osteoarthritis in X-rays [27]; motion restriction of the affected knee in the sagittal plane; history of neurological, rheumatic and other musculoskeletal diseases in the lower limbs and pain in the lower back, hip, and sacroiliac region [32]; history of vertigo, uncorrected vision problems and inner ear disorders [18]; injection of corticosteroids in the past three months; and taking painkillers in the last 72 hours [34].

To evaluate the participants' dynamic balance, we employed the Y-balance test before and after the intervention (ICC $=0.84-0.92)$. In this test, the subject stands barefoot on one leg in the center and tries to reach three directions of anterior, posterolateral, and posteromedial (Figure 1). The test was repeated three times in each direction, and the maximum obtained numerical value in each direction was recorded [36]. The rest time between each repetition in each direction was 10 seconds, and between each movement was 20 seconds [37]. The following formula was used to eliminate the effect of individual differences such as height on the maximum reach distance:

\section{Score $=$ Reach distance/Limb length $\times 100$}

After 5 minutes of rest and initial tests, the intervention was started. At this stage, TEMTEX kinesio tape with a tension equal to $50 \%-75 \%$ of its initial length was applied on the patella of the involved limb to cause medial glide [38]. The subject was asked to lie on the bed with a straight knee. The tape was measured on the subject's patella and cut into a Y shape. When the subject's knee was straight or bent 20-30 degrees, 


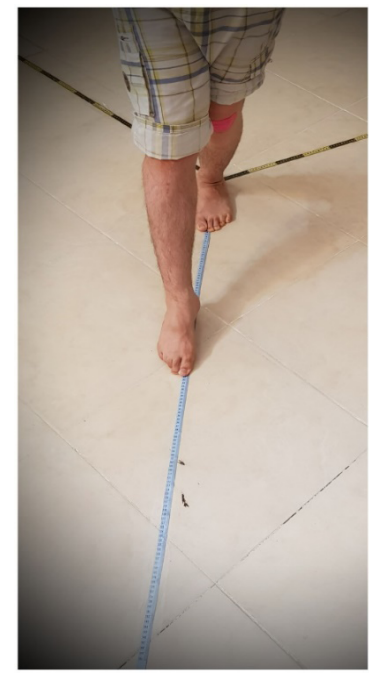

Figure 1. Y-balance test

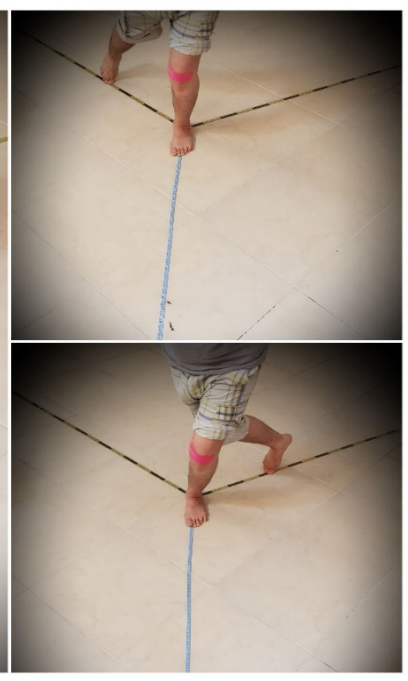

Rehabilitation the first $2 \mathrm{~cm}$ of the tape was attached without stretching to the outside of the patella. The therapist then held the tapeapplied site tightly with one hand and, while simultaneously performing medial gliding over the patella, guided the base of the tape with 50\%-75\% tension towards the knee. During the procedure, the person's knee was bent completely, and the tape base was ended up to the mid-part of the patella. By maintaining the base of the tape, the first tail of the tape was attached to the upper part of the patella with a tension equal to $15 \%-25 \%$ of the initial length, and the second tail was attached to the lower part of the patella with the same amount of tension. Finally, the tail ends were attached to the patella without additional tension (Figure 2) [38].

The effectiveness of kinesio taping was measured by performing a single-leg squat-test. For this purpose, before taping, the person was squatted on the involved leg for 10 seconds with the knee bent 45 degrees, and the pain intensity was recorded by the visual analog scale $(\mathrm{ICC}=0.6)$. This assessment was repeated after taping, and pain intensity before and after taping was finally compared. If the pain were reduced by approximately $50 \%$, the subjects would enter the final stage of the test. Otherwise, the taping would be repeated to achieve the desired pain relief [16]. In the final step, the subjects performed the Y-balance test again. The paired t-test was used for within-group comparison of the changes, and the independent t-test was used to compare the changes between the male and female subjects. The homogeneity of the two groups was examined by Levene's test. The significance level was set at 0.05 .

\section{Results}

The age and anthropometric characteristics of the participants are shown in Table 1 . There was no statistically

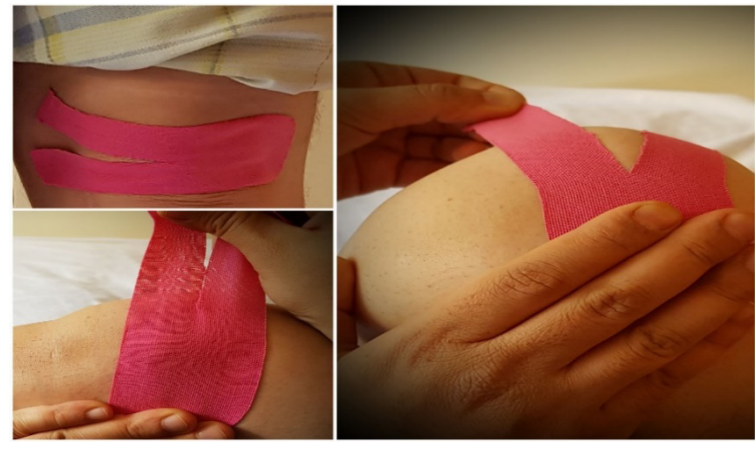

Rehabilitation

Figure 2. Kinesio taping of the patella to correct its external glide

significant difference between male and female patients in age and body mass index based on the independent t-test results $(\mathrm{P}>0.05)$, and the homogeneity of the two groups was proved by Levene's test $(\mathrm{P}>0.05)$. To control the possible effects of anthropometric characteristics, we matched the anthropometric characteristics of men and women. The result of within-group comparison of Y-balance test parameters before and after the kinesio taping in patients using paired $\mathrm{t}$-test showed the highest numerical value of the distance reach in three directions, indicating the significant effect of kinesio taping over patellar on improving balance $(\mathrm{P}<0.05)$ (Table 2). According to the independent t-test, no significant difference was observed in the balance parameters between males and females at baseline $(\mathrm{P}>0.05)$ (Table 2). Moreover, in both male and female groups, the amount of pain was significantly reduced after kinesio taping based on the paired t-test results $(\mathrm{P}<0.05)$, but the between-group comparison of pain intensity showed no significant difference $(\mathrm{P}>0.05)$. MANCOVA was used to compare better the two gender groups and eliminate the effect of pre-intervention scores. To eliminate the effect of the height factor on the reach distance, we normalized the values and reported them in Table 2.

\section{Discussion and Conclusion}

The present study results showed a significant improvement in dynamic balance and reduced the pain of the patients with PFPS before and after kinesio taping, but there was no significant difference between the men and women after kinesio taping. To control the balance system, afferents of proprioceptive, visual, and vestibular receptors must work adequately [39-41]. The impairment of these systems can impair balance [40]. Proprioceptive information obtained from mechanical receptors in muscles, joints, and ligaments is the most important component of somatosensory afferents and has a vital role in maintaining joint function and balance control [59]. In many studies, proprioception disorder in individuals with PFPS has been reported $[9,21]$. It has been reported that knee joint proprioception, 
Table 1. Age and anthropometric characteristics of the participants

\begin{tabular}{cccc}
\hline Characteristics & \multicolumn{2}{c}{ Mean \pm SD } & P \\
\cline { 2 - 3 } & Women $(\mathbf{n}=\mathbf{3 1})$ & Men $(\mathbf{n}=\mathbf{3 0})$ & $<0.001$ \\
\hline Height $(\mathrm{cm})$ & $162.3 \pm 6.5$ & $174.9 \pm 6.6$ & $<0.001$ \\
\hline Weight $(\mathrm{kg})$ & $71.03 \pm 10.98$ & $81.60 \pm 1.73$ & $<0.869$ \\
\hline Body mass index $\left(\mathrm{kg} / \mathrm{m}^{2}\right)$ & $26.9 \pm 3.51$ & $26.74 \pm 4.038$ & $<0.402$ \\
\hline Age $(\mathrm{y})$ & $34.77 \pm 5.78$ & $33.53 \pm 5.67$ & \\
\hline
\end{tabular}

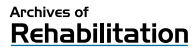

which is essential in creating balance, is weaker in people with PFPS than in healthy people. Therefore, it seems that impairment in proprioception affects the body's motor control and decreases balance [42]. Given the crucial role of proprioception and muscle activity for balance and their impairment in individuals with PFPS, balance disorders in these patients are expected [15].

Of course, the role of pain should not be ignored in the outcome. Patellofemoral joint reaction force in people with PFPS is more common, which can cause pain during functional activities [43]. Pain may inhibit the muscles around the knee [44]. Since these muscles are responsible for timely and effective motor responses in controlling the condition of the body, the impact of kinesio taping on improving the balance of individuals with PFPS may be related to its effect on the systems that affect the balance and reduce pain [44, 45]. The effect of kinesio taping on cutaneous mechanoreceptors and improvement of knee joint proprioception has already been reported $[19,46,47]$. Since sensory inputs can increase central nervous system feedback and reduce pain, the effect of kinesio taping seems to be justified based on the gate theory of pain. Considering the significant reduction of pain in all subjects in our study after the intervention and considering the direct relationship between pain and postural fluctuations, the improvement of balance can be justified [4]. Reduced pain increases the activity of the vastus medialis oblique muscle and helps stabilize the knee joint during testing [27]. As a result, it allows people to balance more effectively as they try to reach a greater distance. Because the

Table 2. Comparing the Y-balance test results between men and women with PFPS

\begin{tabular}{|c|c|c|c|c|c|}
\hline \multirow{2}{*}{ Variable } & \multirow{2}{*}{ Group } & \multicolumn{3}{|c|}{ Mean \pm SD } & \multirow{2}{*}{$\mathbf{P}$} \\
\hline & & Before the Intervention & After the Intervention & Mean Difference & \\
\hline \multirow{3}{*}{ Anterior reach distance $(\mathrm{cm})$} & Men & $69.30 \pm 5.63$ & $73.38 \pm 5.61$ & $4.08 \pm 2.64$ & $<0.001^{*}$ \\
\hline & Women & $68.24 \pm 6.59$ & $73.49 \pm 5.97$ & $5.25 \pm 2.87$ & $<0.001^{*}$ \\
\hline & $\mathrm{P}$ & $0.502^{* *}$ & $0.102^{* * *}$ & - & - \\
\hline \multirow{3}{*}{$\begin{array}{l}\text { Posterolateral reach distance } \\
\qquad(\mathrm{cm})\end{array}$} & Men & $69.66 \pm 13.35$ & $76.84 \pm 12.48$ & $7.18 \pm 3.77$ & $<0.001^{*}$ \\
\hline & Women & $66.31 \pm 10.62$ & $75.25 \pm 10.85$ & $8.93 \pm 3.87$ & $<0.001^{*}$ \\
\hline & $\mathrm{P}$ & $0.282^{* *}$ & $0.051^{* * *}$ & - & - \\
\hline \multirow{3}{*}{$\begin{array}{l}\text { Posteromedial reach distance } \\
\qquad(\mathrm{cm})\end{array}$} & Men & $71.13 \pm 13.68$ & $77.03 \pm 12.43$ & $5.90 \pm 3.78$ & $<0.001^{*}$ \\
\hline & Women & $65.73 \pm 10.89$ & $73.49 \pm 11.81$ & $7.75 \pm 5.57$ & $<0.001^{*}$ \\
\hline & $\mathrm{P}$ & $0.093^{* *}$ & $0.051^{* * *}$ & - & - \\
\hline \multirow{3}{*}{ Pain } & Men & $5.93 \pm 2.06$ & $2.38 \pm 1.22$ & $3.55 \pm 1.51$ & $<0.001^{*}$ \\
\hline & Women & $6.2 \pm 1.77$ & $2.21 \pm 1.31$ & $3.99 \pm 1.39$ & $<0.001^{*}$ \\
\hline & $\mathrm{P}$ & $0.585^{* *}$ & $0.264^{* *}$ & - & - \\
\hline
\end{tabular}


muscles around the knee need to function adequately and without delay to maintain balance, delayed muscle activity in people with PFPS leads to dysfunction of the sensorimotor system for controlling postural stability and increased risk of losing balance [17]. Patellar taping improves patellar proprioception and stability, leading to improved knee function [19]. Because of the close relationship between the sensory and motor control systems, studies have shown an improvement in neuromuscular activity following the use of tapes [19, 29, 48, 59]. It seems that the improvement in proprioception after kinesio taping is due to the feedback increase transmitted from the skin, soft tissue, ligaments, and muscles to the upper nerve centers [50]. Patellar taping alters afferents and affects the ability to maintain dynamic postural control in people with PFPS [27]. According to the present study results and the relationship between pain reduction and balance improvement [51], the increased reach distance in the specified directions during the Y-balance test (improved dynamic balance) seems reasonable.

In the present study, despite the anthropometric differences between men and women with PFPS, there was no significant difference between them in terms of maximum reach distance and dynamic balance after kinesio taping. The nonsignificant difference in pain between men and women (despite its decreasing trend after the intervention) is probably related to the negligible effect of kinesio taping on women and men's balance with PFPS. Hence, kinesio taping may affect the dynamic balance of men and women with PFPS. Therefore, gender does not affect kinesio taping. Although proprioception was not examined in this study, the increase in reach distance may be due to stimulation of mechanoreceptors and the effect of proprioception. Besides, there are the effects of reduction in pain and improvement in vastus medialis oblique muscle activity [19, 27].

Kinesio taping has the same effect on the dynamic balance of women and men with PFPS. Patellar kinesio taping is recommended as a suitable treatment method for reducing pain and improving dynamic balance in patients with PFPS.

\section{Ethical Considerations}

Compliance with ethical guidelines

This study was approved by the Ethics Committee of Isfahan University of Medical Sciences (code: RESEARCH. REC.1397.040) and registered in the Iranian Registry of Clinical Trials (code: IRCT20181001041197N1). Before the study, the research objectives and method were explained to the participants, and written informed consent was obtained. They were free to leave the study at any time and were assured of the confidentiality of their information.
Funding

This study was extracted from the Master's thesis of the last author, Department of Epidemiology and Statistics, School of Health, Isfahan University of Medical Science, Isfahan. The study received financial support from the Deputy for Research and Technology of Isfahan University of Medical Sciences.

\section{Authors' contributions}

Study design and ideation: Javid Mostamand, Zohreh Shafiezadegan, Zeinab Sadat Hosseini; Data collection: Zeinab Sadat Hosseini; Data analysis and interpretation: Mohammad Javad Tarahi, Zeinab Sadat Hosseini, Zohreh Shafiezadegan and Javid Mostamand; Manuscript edited by: Zeinab Sadat Hosseini; Expert evaluation of the manuscript in terms of scientific concepts and approval of the final manuscript: Javid Mostamand and Zohreh Shafiezadegan. Maintaining the integrity of the study process from the beginning to the publication and responding to the opinions of the judges: Javid Mostamand.

\section{Conflict of interest}

The authors declared no conflict of interest.

\section{Acknowledgments}

The authors would like to thank Dr. Khadem, Physiotherapy Clinic of Ayatollah Sadoughi Hospital in Isfahan City, and all participants for their valuable cooperation. 


\title{
مقايسه تأثير خسب كينزيولوزى روى تعادل يويا و درد بين زنان و مردان مبتلا به سندرم درد كشككى رانى يكىطرفه تاتير يسب
}

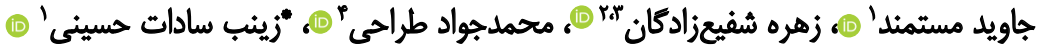 \\ ا. كروه فيزيوثرابي، مركز تحقيقات اختلالات اسكلتى و عضلانى، انشكده علوم توانبخشى، دانشعاه علوميزشكى اصفهان، اصفهان، ايران.

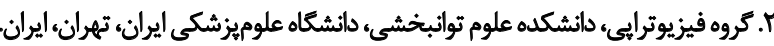

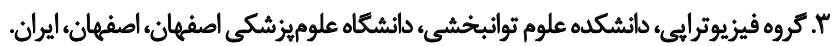

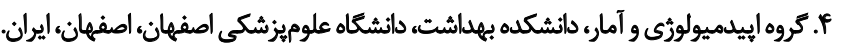

\begin{abstract}
حكبد

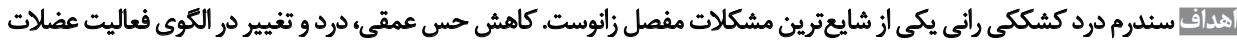

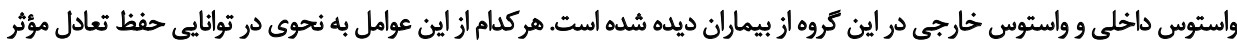

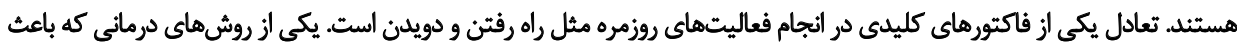

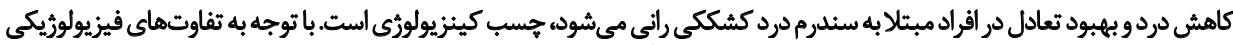

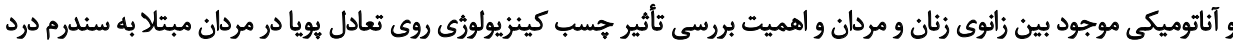

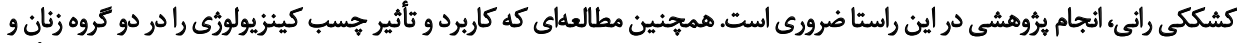

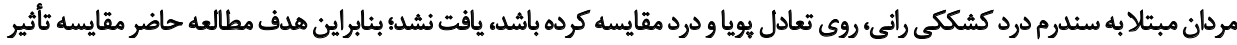

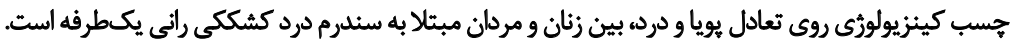

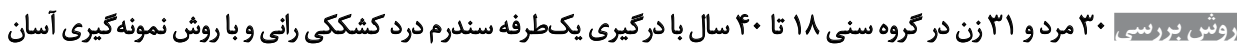

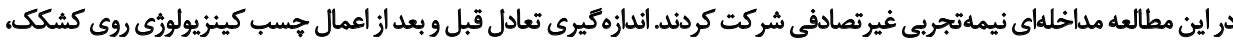

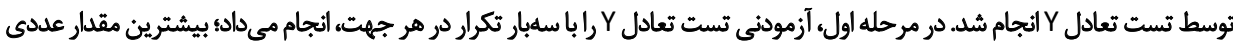

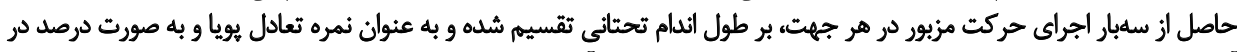

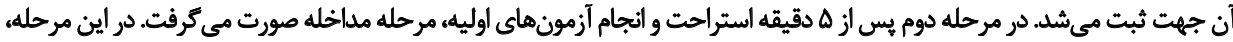

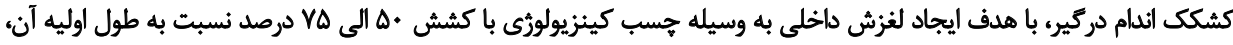

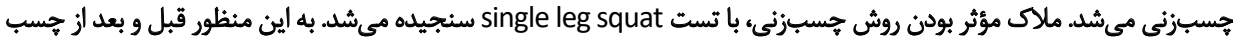

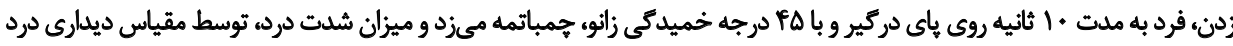

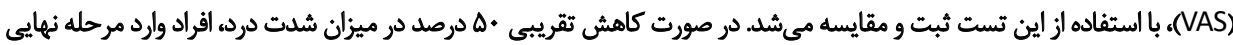

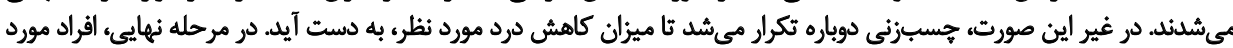

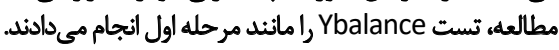

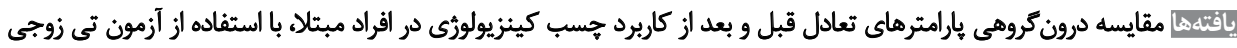

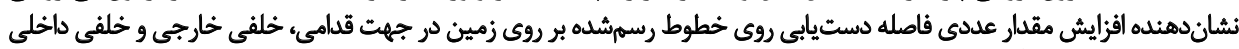

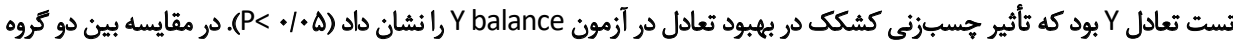

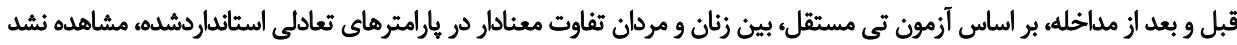

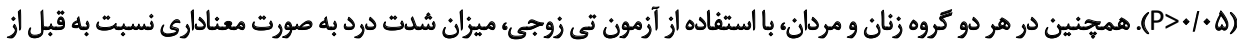

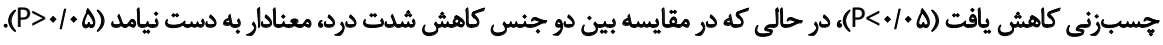

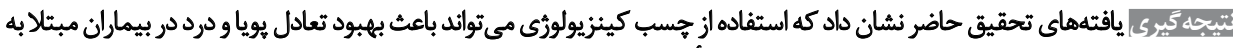

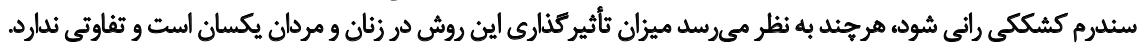

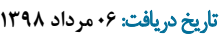
تاريخ هذيرش: 19 مرداد 
جمعيت كر فتتار به اين سندرم، درمان موفق از طريق فيزيوترايى

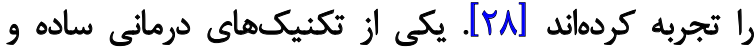

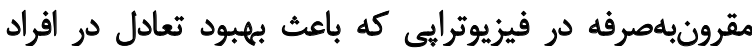

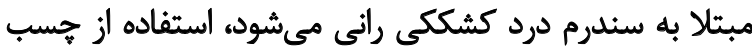

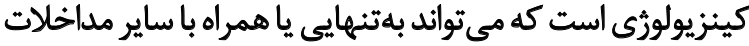

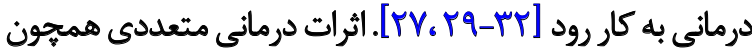

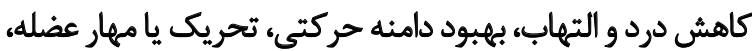

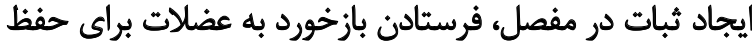

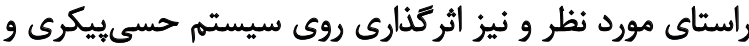

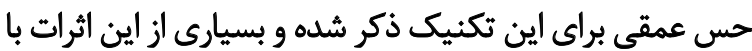

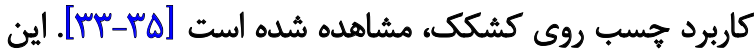

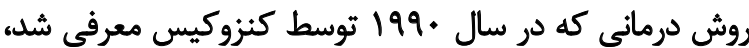

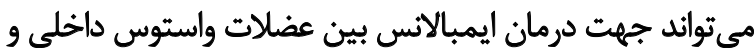

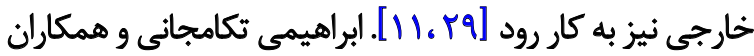

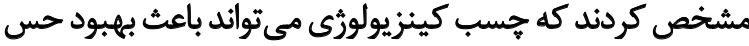

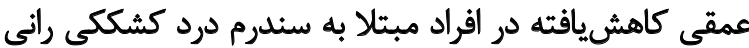

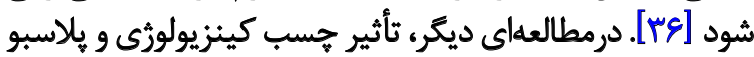

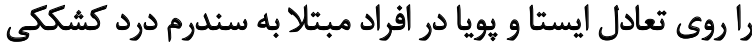

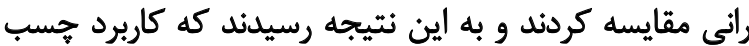

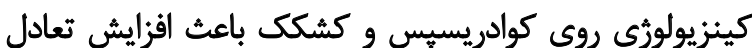

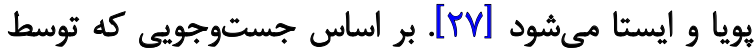

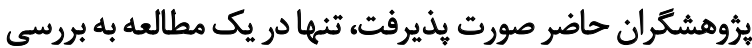

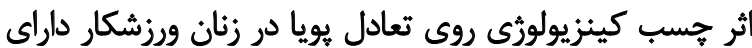

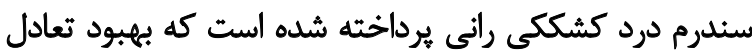

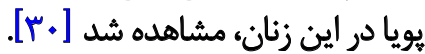
با توجه به اينكه جسب كينزيولورثى نسبت به مئ مك كانل

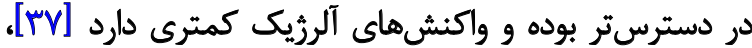

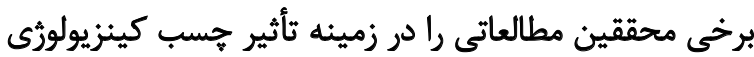

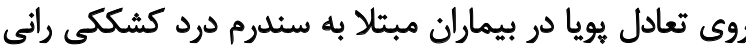

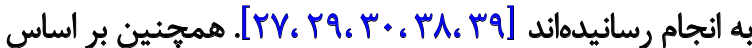

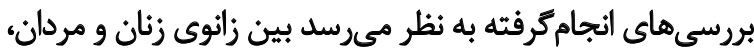

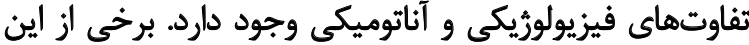

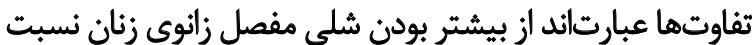

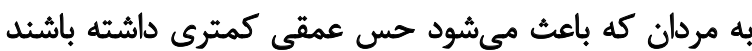

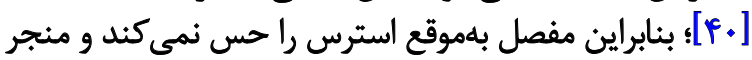

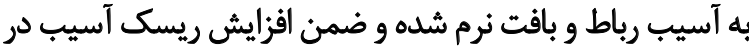

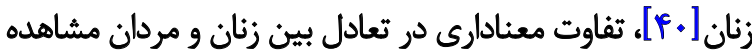

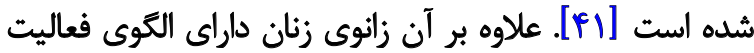

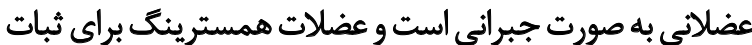

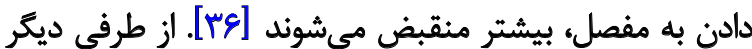

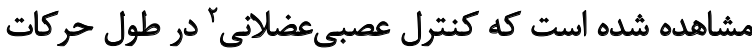
دايناميك و فعاليتهاى ورزشى و فيزيكى بين نوجوانان مؤنث
مقلمه

وجود درد در قسمت قدامى و خلفى كشكك، بدون حضور

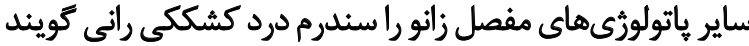

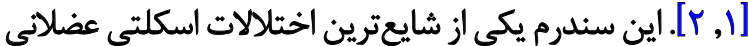

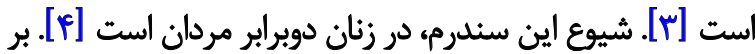

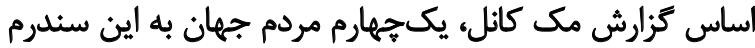

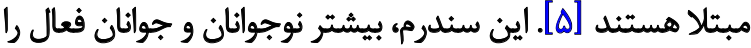

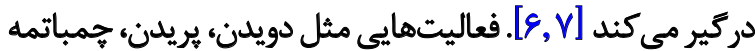

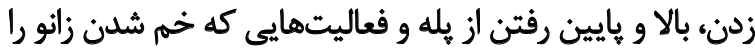

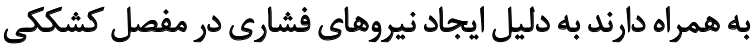

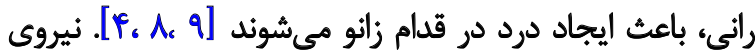

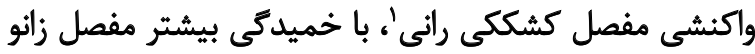

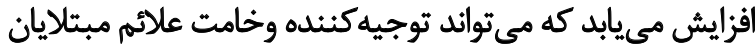

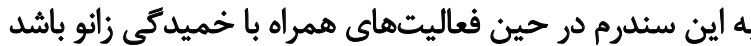

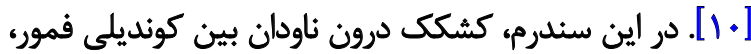

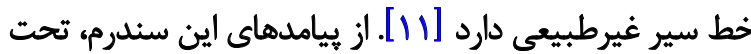
تأثير قرار كرفتن كنترل تعادل (حفظ موقعيت در وضعيت ايستا لإينا

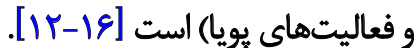

تعادل نيازى اساسى براى انجام فعاليتهاى روزمره است و

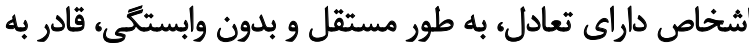

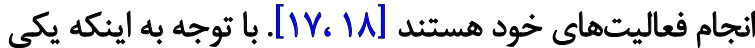

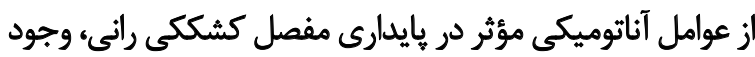

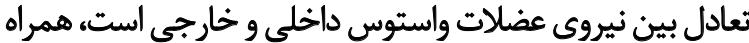

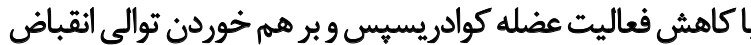

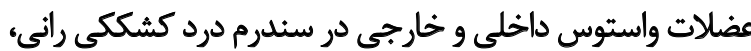

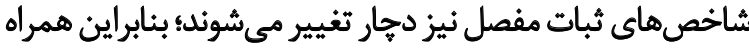

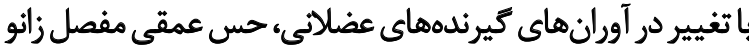

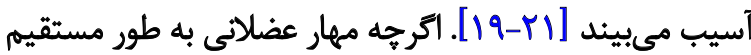

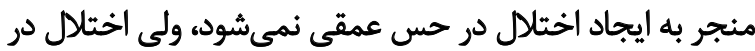

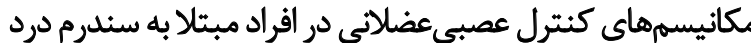

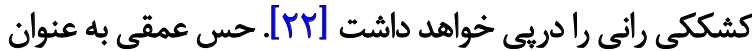

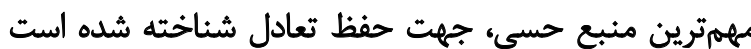

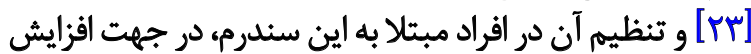

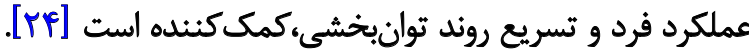

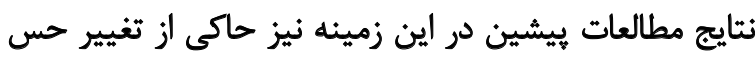

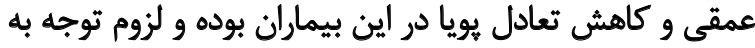

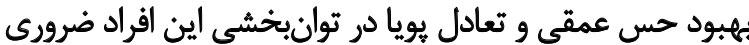

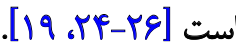

با توجه به خطر ابتلابه استئوآرتريت، درمان صحيح و زودهنكام

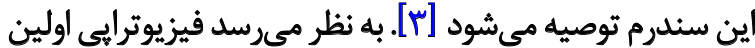

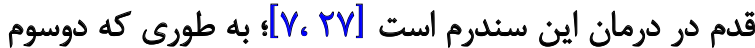




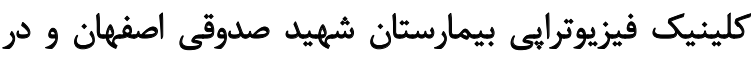

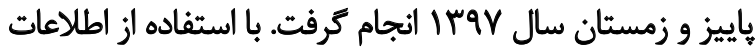

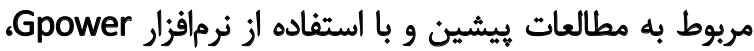

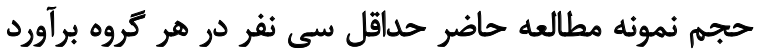

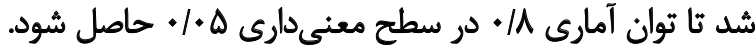

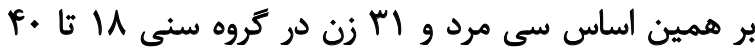

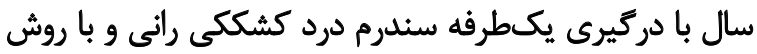

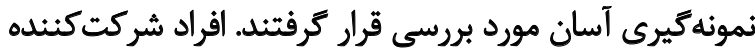

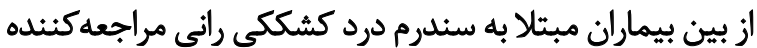

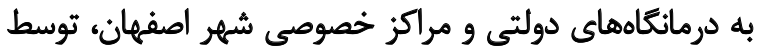

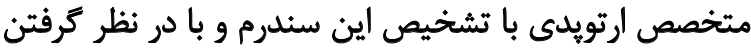

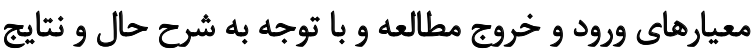

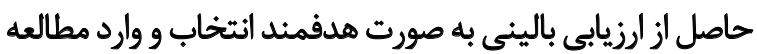

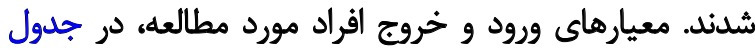

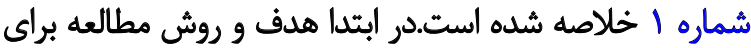

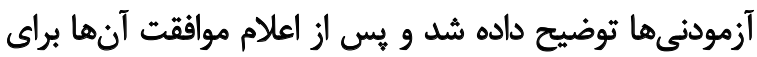

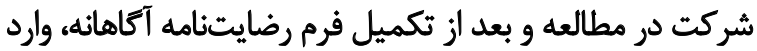

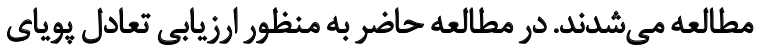
شركت كنيدكان از تست ديت

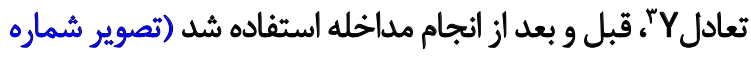

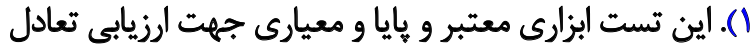

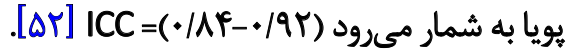

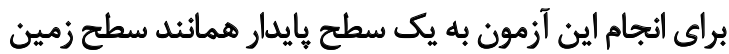

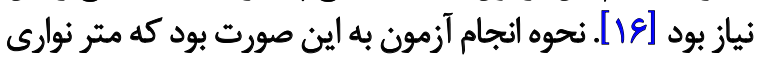

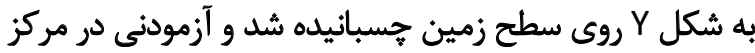

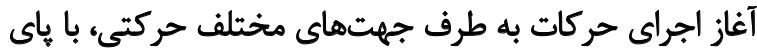

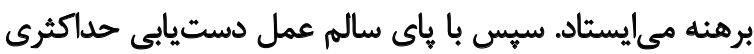

\section{Y Balance Test}

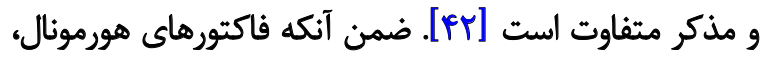

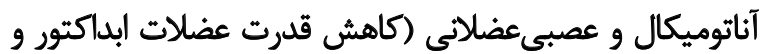

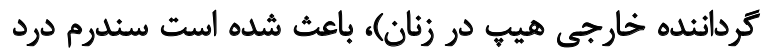

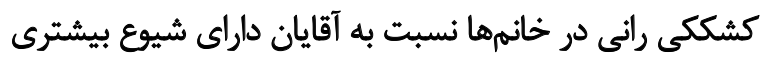

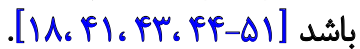
بر اساس جستوجوى يرؤششكران حاضر، يرؤهشى كه تأثير

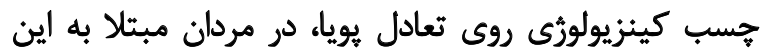

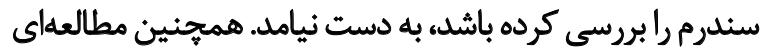

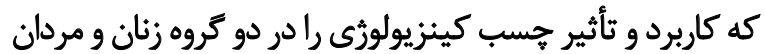

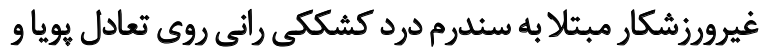

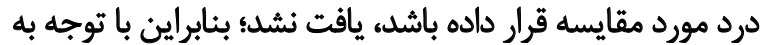

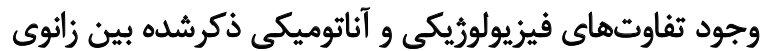

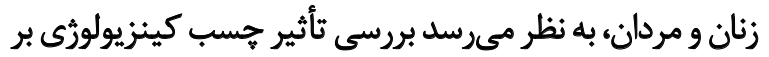

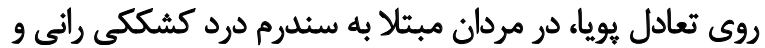

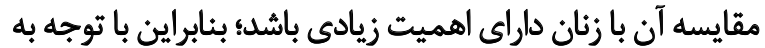

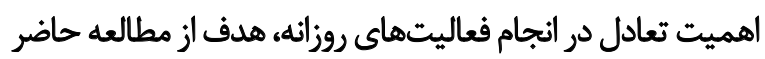

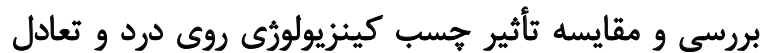

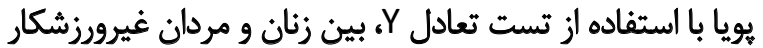

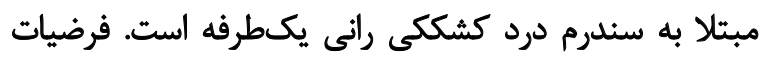

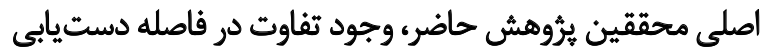

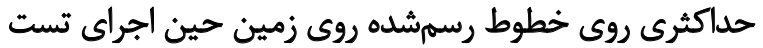

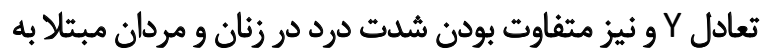

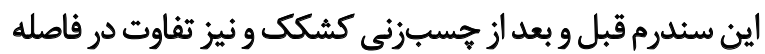
دستيابى و ميزان درد بين دو جنس بودي

$$
\text { روش بروسى }
$$

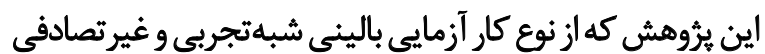

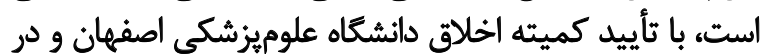

جدول (. معيارهاى ورود وخروج

\section{معيارهاى ورود وخروج}

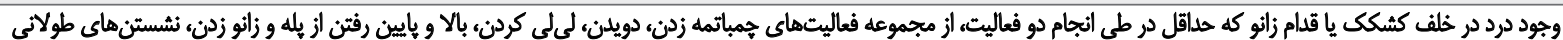

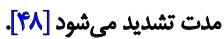

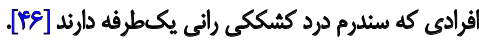

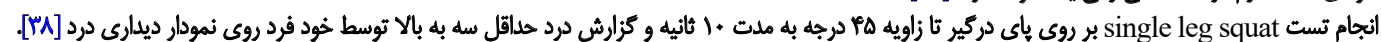

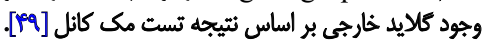

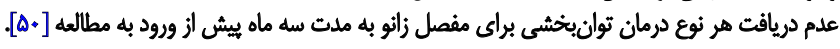

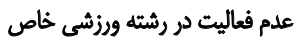

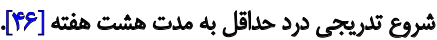

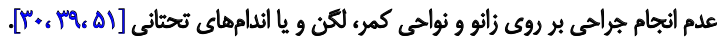

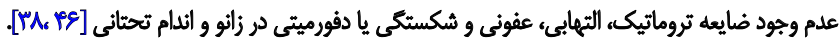

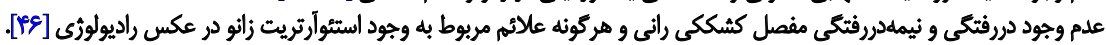

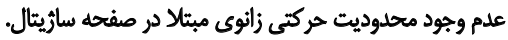

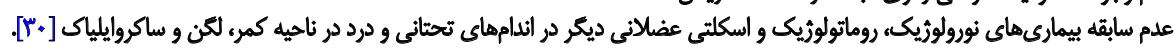

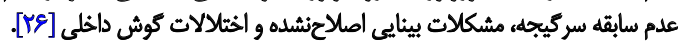

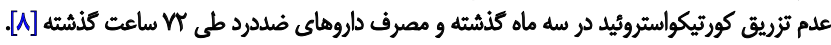




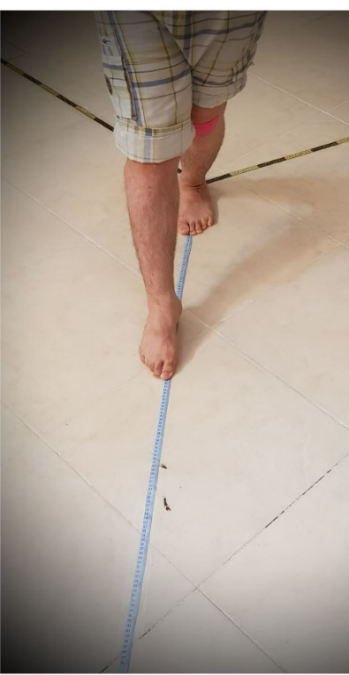

توانبخننى

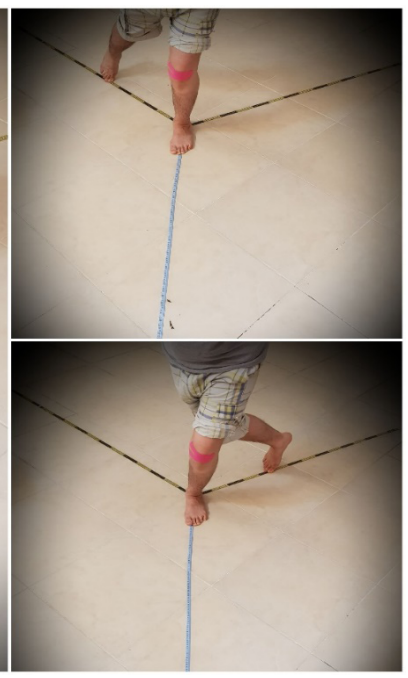

تصوير ب. روش انجام تست تعادل Y

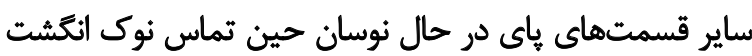

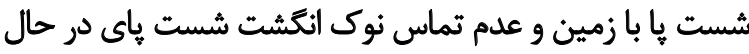

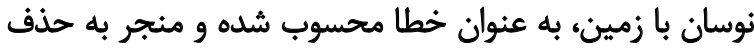

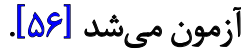

نوماليزأسيون

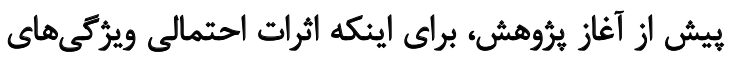

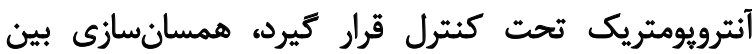

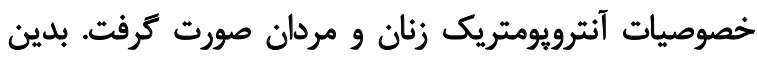

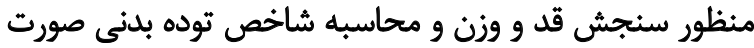

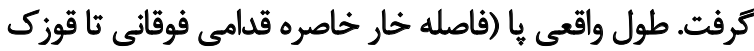

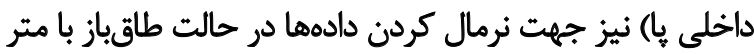

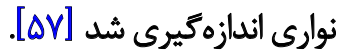

مداخله (kinesio tape)

بس از ه دقيقه استراحت و انجام آزمونهاى اوليه، مرحله

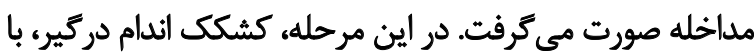

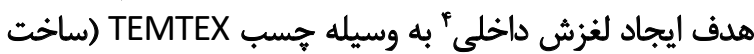

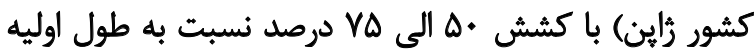

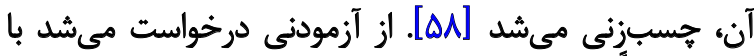

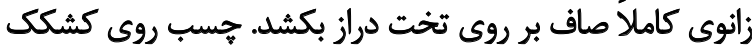

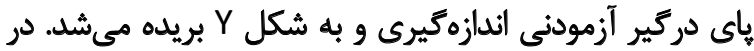

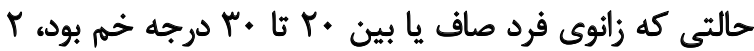

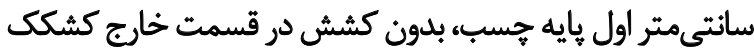

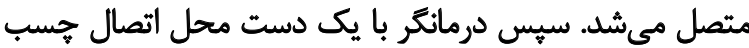

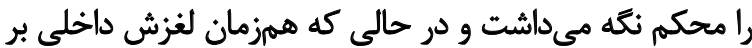

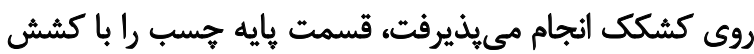

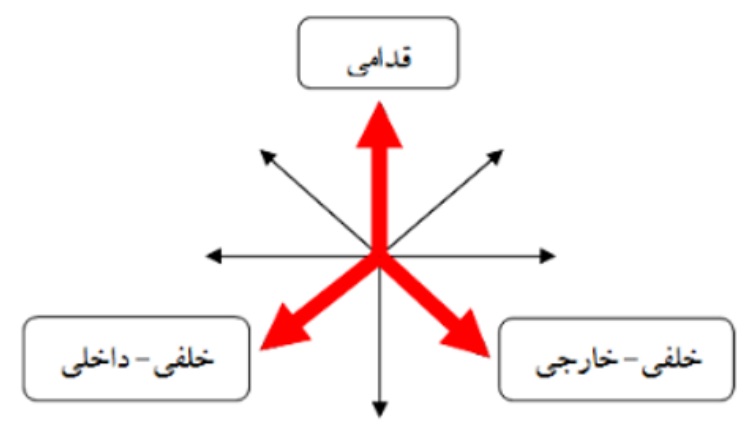

توانبخننى

رورى خطوط رسمشده بر زمين را در جهات قدامى، خلفى

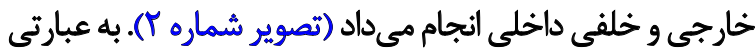

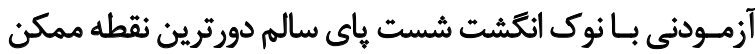

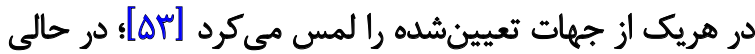
كه باى مبتلا به سندرم درد كشككى رانى در مركي مركز

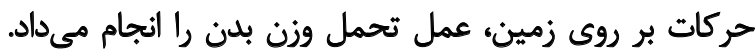

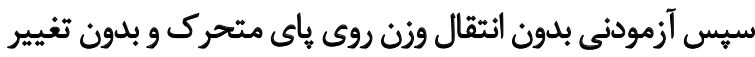

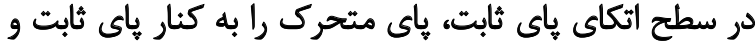

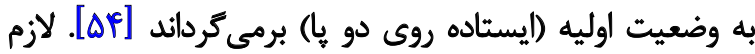

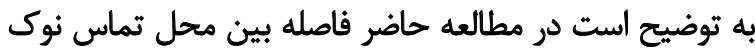

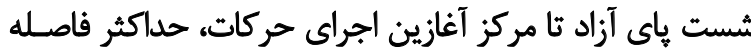

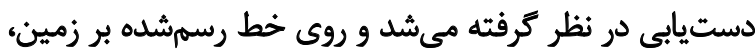

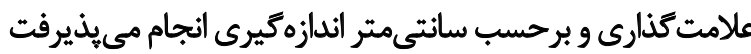

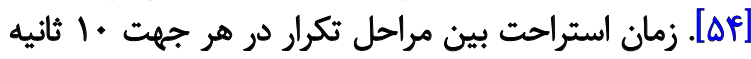

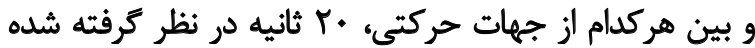

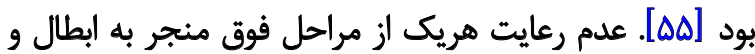

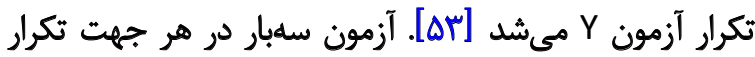

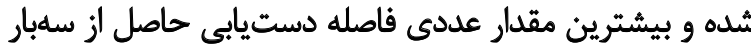

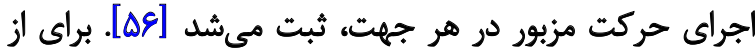

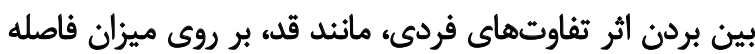

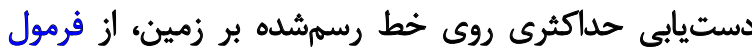

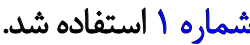

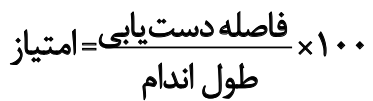

بر اساس اين فرمول بيشترين مقدار عددى فاصله دستيابى بردي

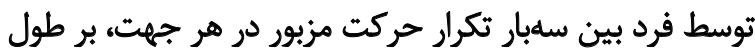

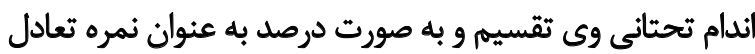
يويا در آن جهت ثبت مىشد تصند

به منظور به حداقل رساندن اثرات يادكيرى، جهت جلوكيرى

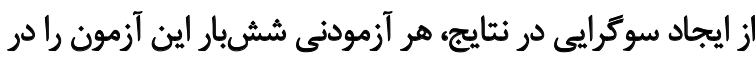

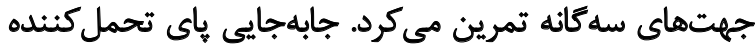

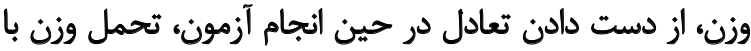




\section{يافتهها}

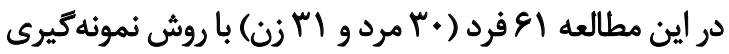

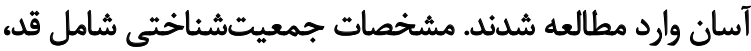

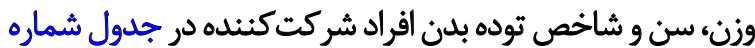

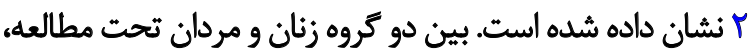

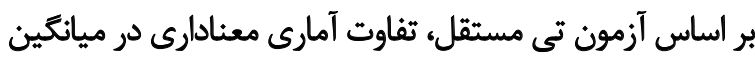

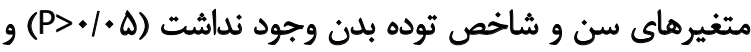

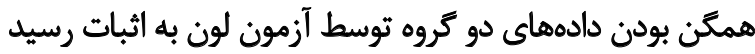

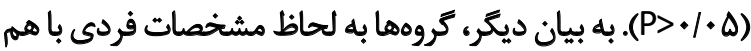

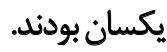

نتيجه مقايسه درون كروهى يارامترهاى تست تعادل لاقبل و بعد

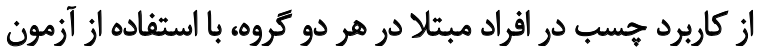

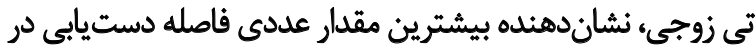

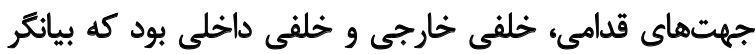

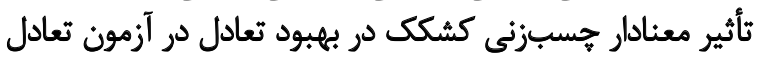

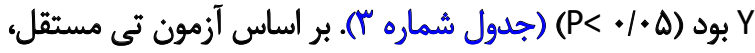

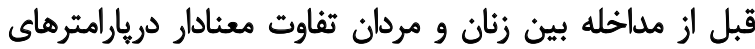

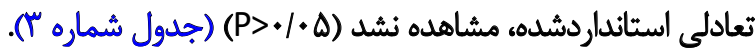

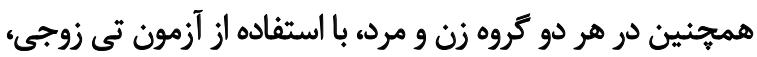

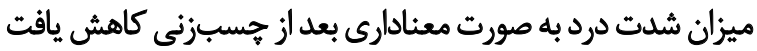

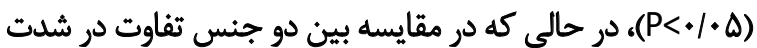

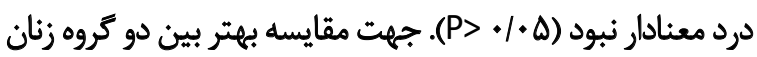

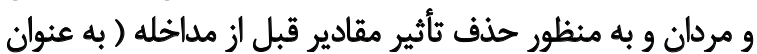

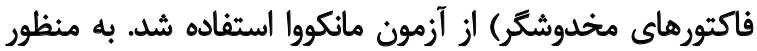

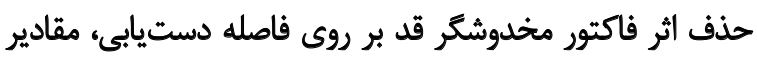

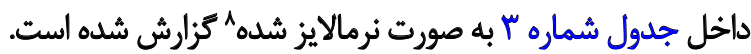

بحث

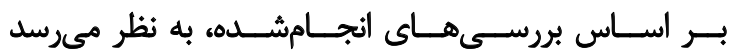

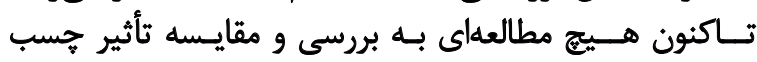

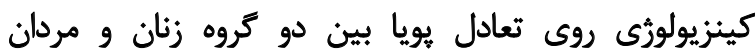

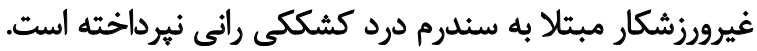

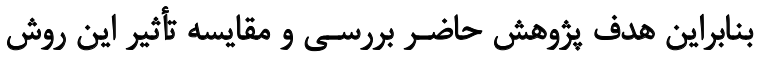

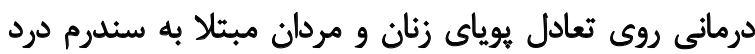

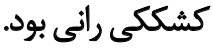

فرضيات اصلى مطالعه حاضر اين بود كه ميزان فاصله

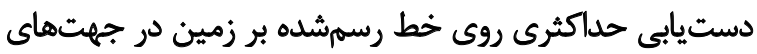

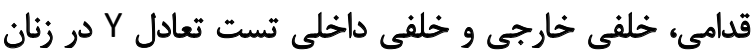

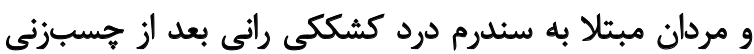

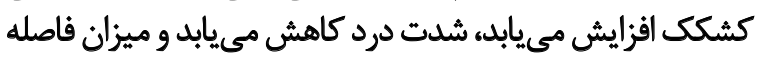
دستيابى و شدت درد بين زنان و مردان نيز متفاوت است دئ نتيجه

\section{Normalized}

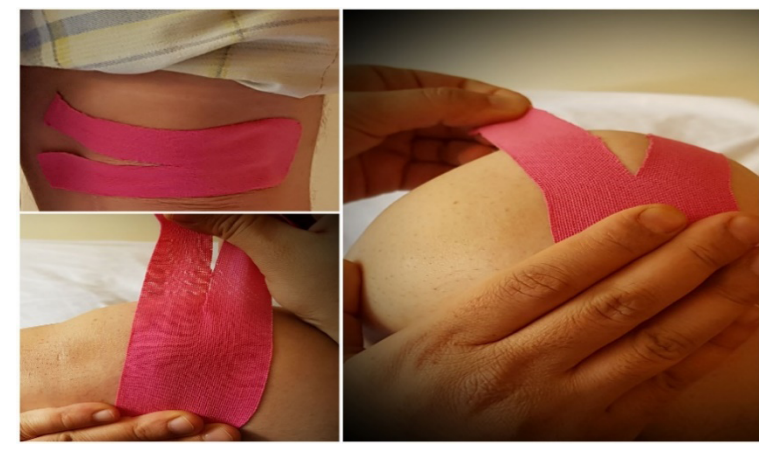

توانبخننى

تصوير "ا. نحوه هسبزنى كشكك به منظور اصلاح لغزش خارجى آن

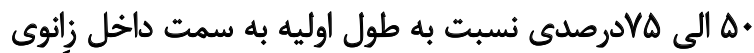

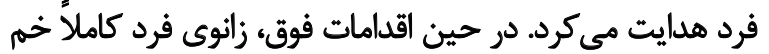

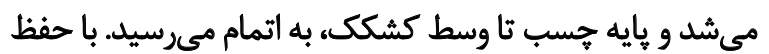

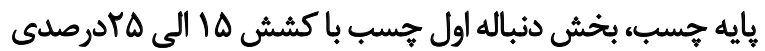

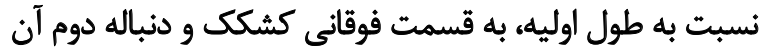

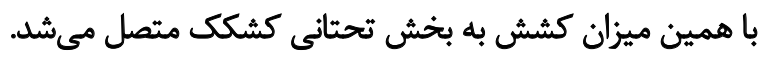

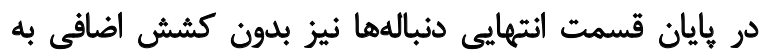

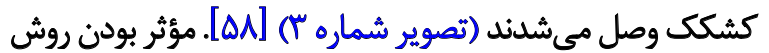

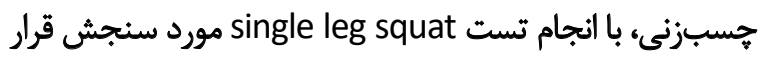
مي

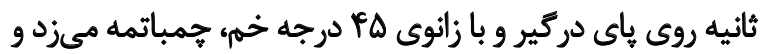

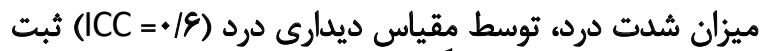

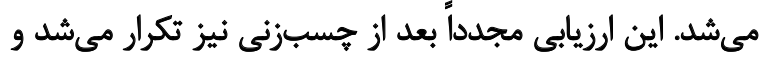

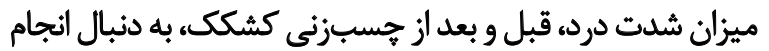

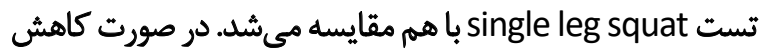

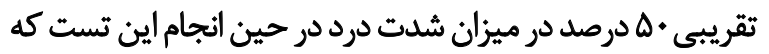

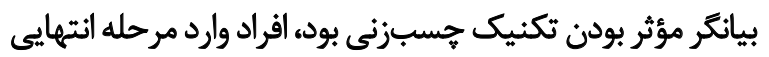

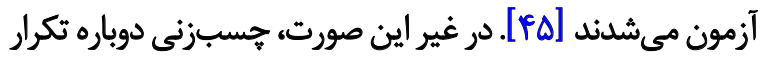

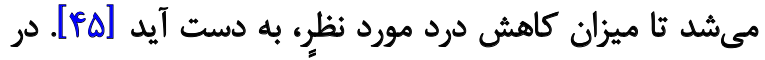

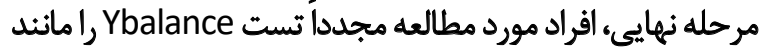
مرحله اول انجام مي دادند.

با توجه به نرمال بودن توزيع دادهها بر اساس آزمون كولموكروف

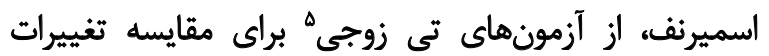

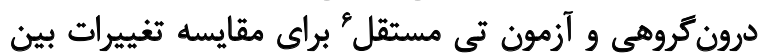

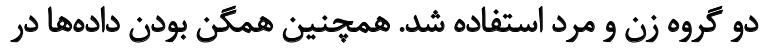

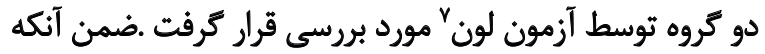

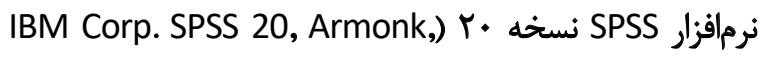
و و با سطح معنى دارى ه • ٪ براى تحليل دادهها مورد استفاده قرار ترفت.

5. Paired t-test

6. Independent t-test

7. Levene's Test 
جدول r. مقايسه ميانكين قده وزن، نمايه توده بلن و سن زنان و مردان تحت مطالعه مبتلا به سندرم درد كشككى رائى

\begin{tabular}{|c|c|c|c|}
\hline \multirow{2}{*}{$\mathbf{P}$} & \multicolumn{2}{|c|}{ ميانكين=|نحراف معيار } & \multirow{2}{*}{ متغير } \\
\hline & مردان ("rn=r) & زنان (n=rا') & \\
\hline$<+1+\infty 1$ & $1 v e / q_{ \pm} 8 / 8$ & $\mid E Y / T \pm F / \theta$ & قد (سانتى متر) \\
\hline$<+1 *+1$ & $11 / 8 \cdot \pm 1 \cdot / M^{r}$ & $w / . r \pm 1 . / q u$ & ونن (كيلوكرم) \\
\hline$<+\mathbb{1 N}=9$ & $r g / M^{\prime} \pm r / \% \Lambda$ & $r g / q \pm r / \Delta 1$ & شاخص توده بدن (كيلوكرم بر متر مربع) \\
\hline$<\cdot / * \cdot r$ & $\pi / \Delta r \pm \Delta / \& V$ & $M / W \pm \Delta / V A$ & سن سن \\
\hline
\end{tabular}

حفظ و بازگرداندن ثبات عملكردى مفصل تعريف شود [09].

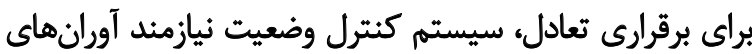

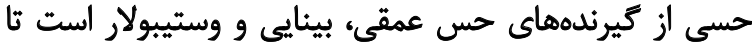

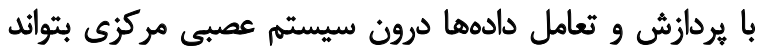

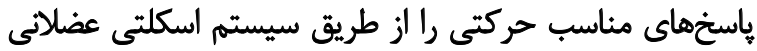

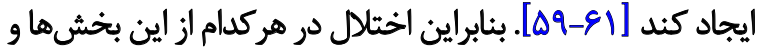

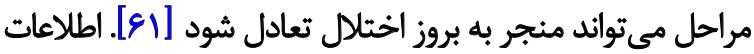

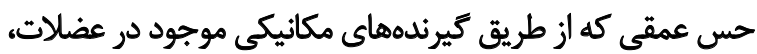

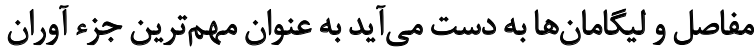

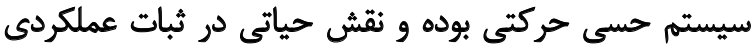

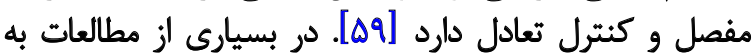

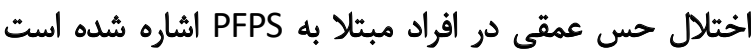

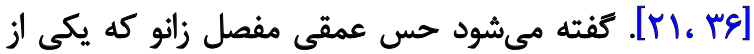

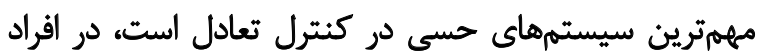

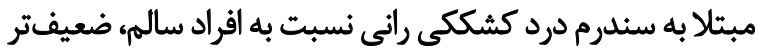

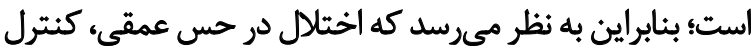

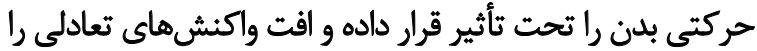

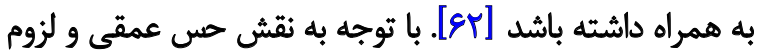

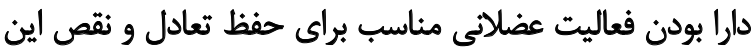

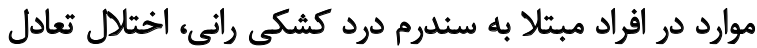

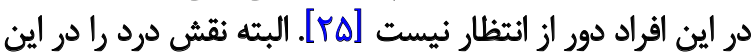

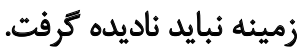

نيروى واكنشى مفصل كشككى رانى بين اقراد سالمو افراد مبتلا

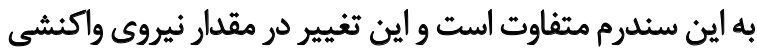

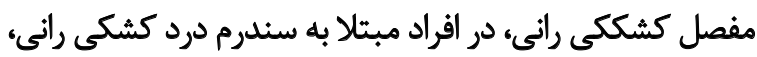

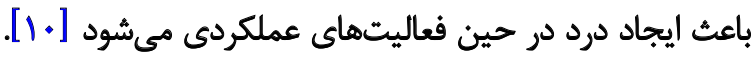

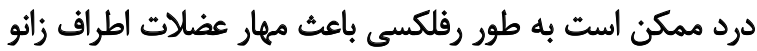

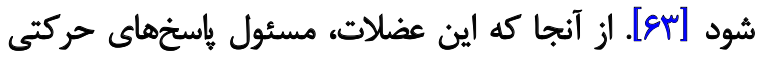

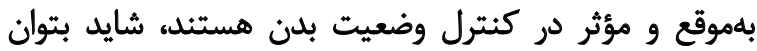

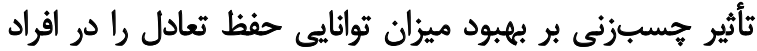

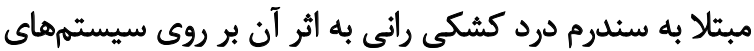

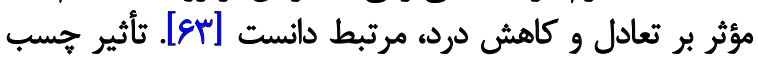

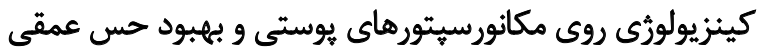

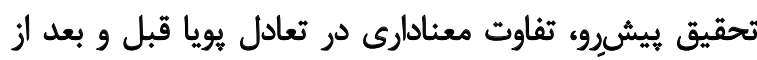

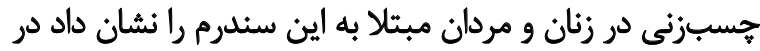

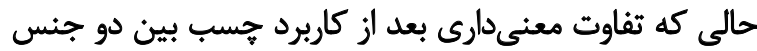

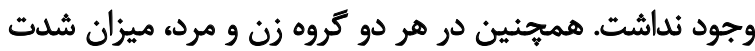

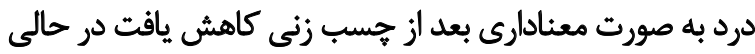

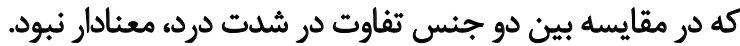

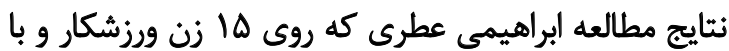

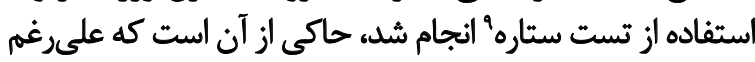

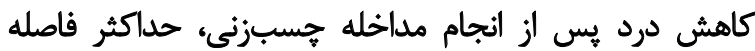

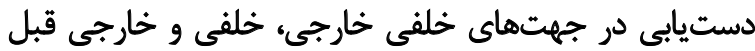

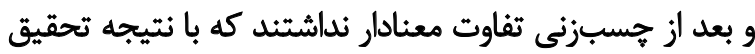

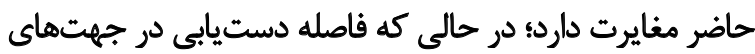

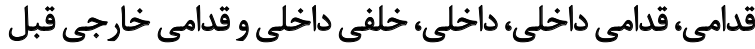

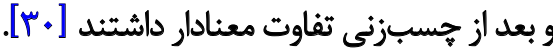

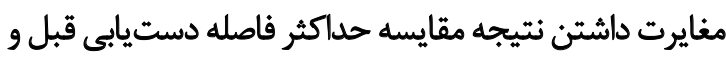

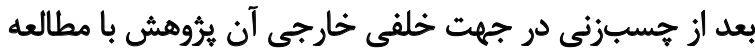

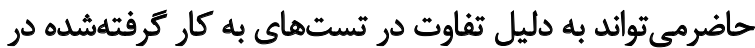

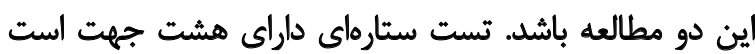

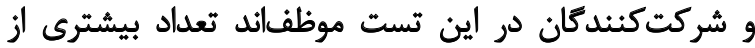

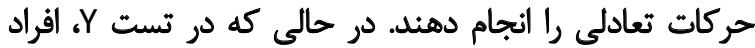

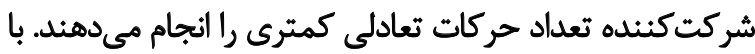

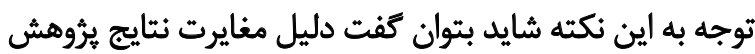

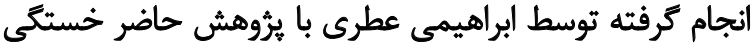

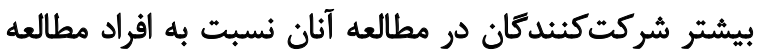

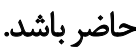

كثترل ياسجر تحت عنوان وجودارتباطى متقابل و يُبيجيده ميان

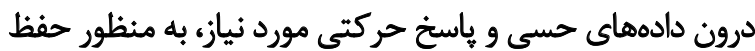

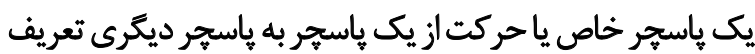

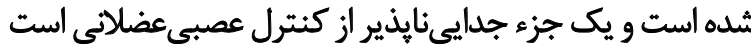

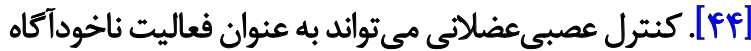
در آمادهسازى و در ياسخ به حركت و باركيرى مفصل به به منظوري

\section{Star Excursion Test}


جدول "ب. مقايسه ميانكين فاصله دستيابى قدامى، خلفى خارجى، خلفى داخلى و نمره درد بين زنان و مردان تحت مطالعه مبتلا به سئدرم درد كشككى رائى

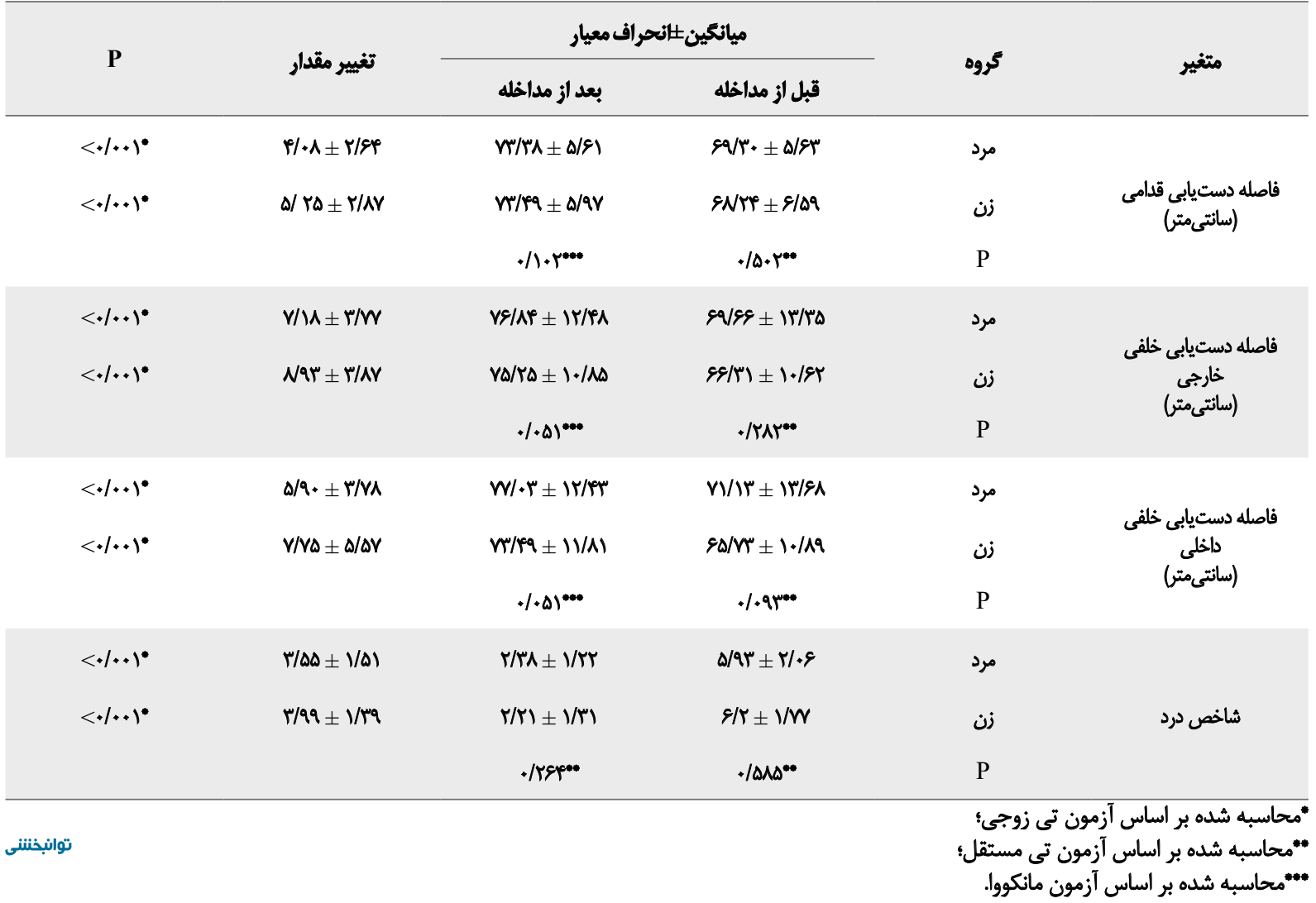

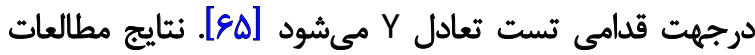

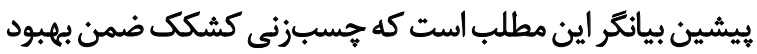

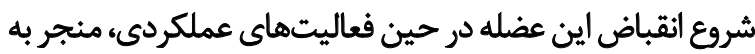

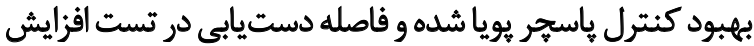

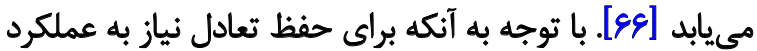

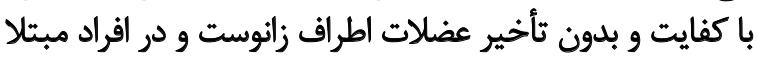

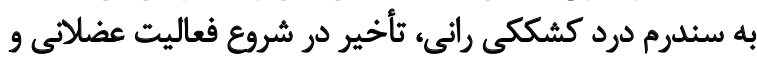

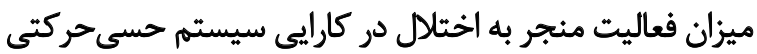

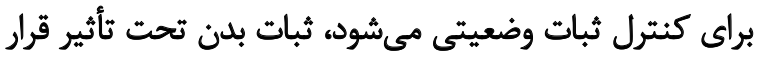

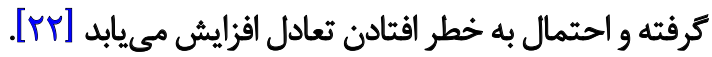

جسبزني كشكك، حس عمقى و ثبات كشكى را ارتقا داده

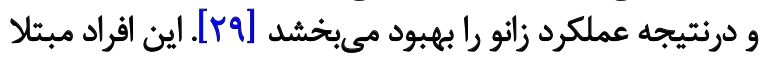

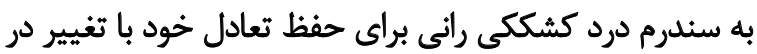

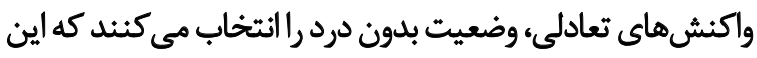

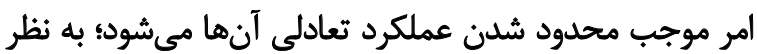

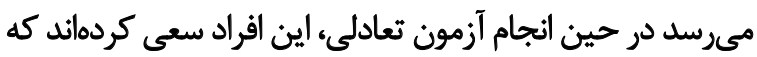

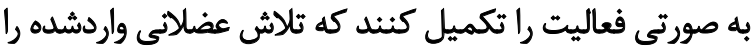

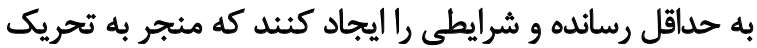

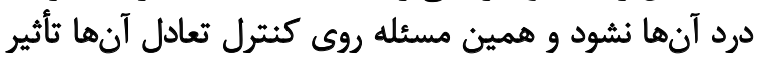

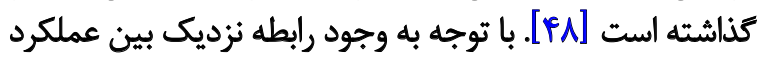

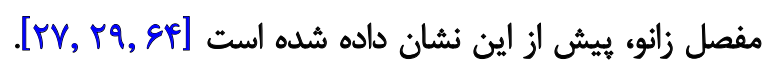

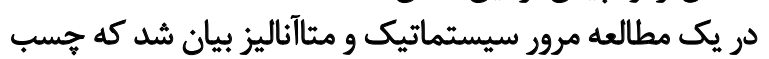

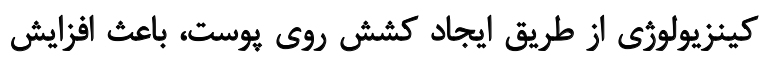

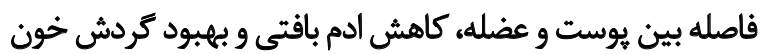

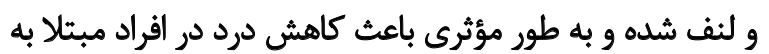

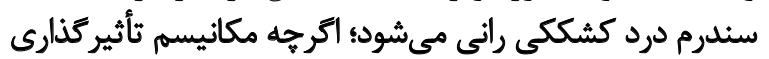

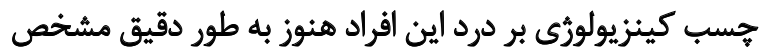

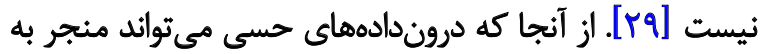

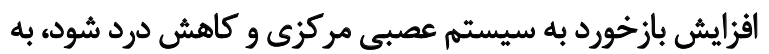

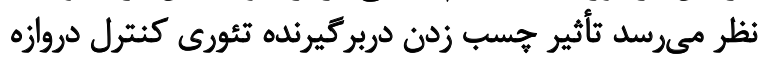

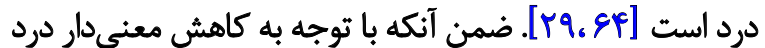

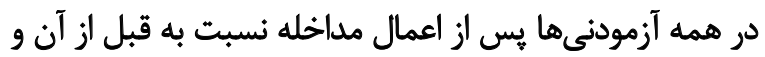

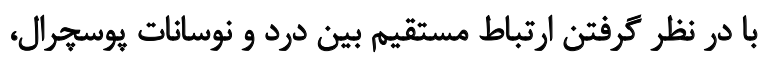

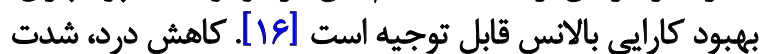

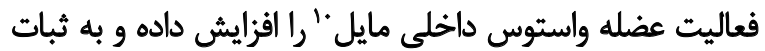

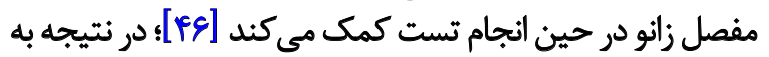

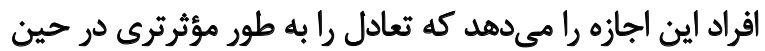

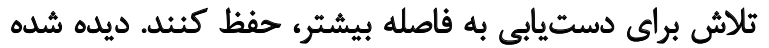

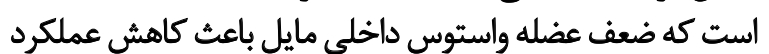

10. Vastus Medialis Obliques (VMO) 
درمائى مناسب براي بهبود درد و تعادل يويا در بيماران مبتلا به ديه

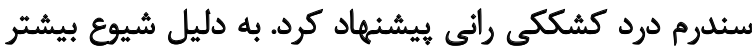

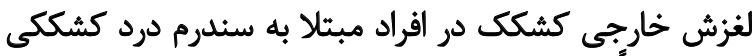

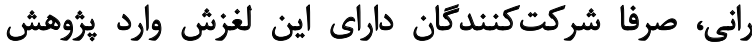

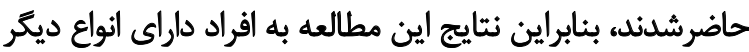

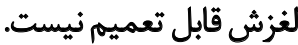

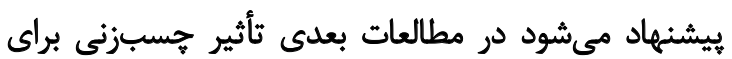

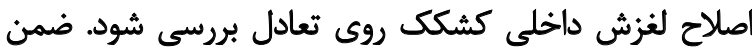

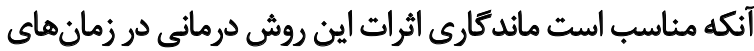

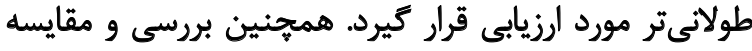

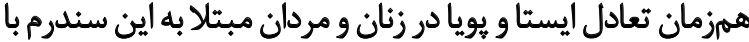

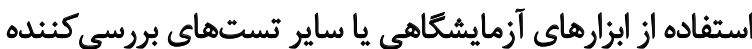
تعادل، بسيار شايسته است.

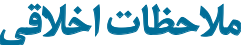 \\ ي ميروى از اصول الخلاق يُوهش}

اين مطالعه با تأييد كميته اخلاق دانشكاه علوميزشكى اصفهان

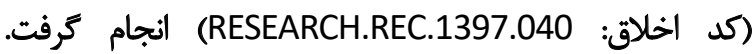
ضـــن آنكه در مركز بين المللى ثبت كار آزمايىهاى بالينى ايران،

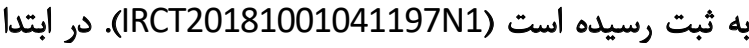

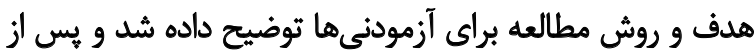

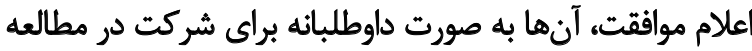

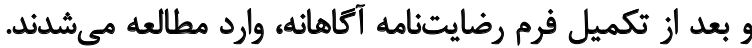

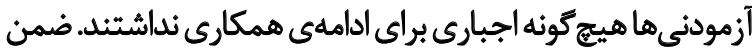

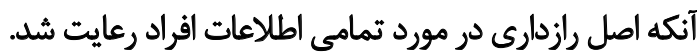

$$
\text { مامي مالب }
$$

اين مقاله مستخرج از إياينامه كارشناسى ارشد نويسنده

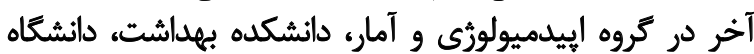

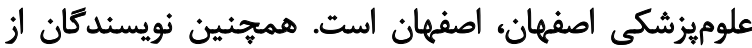

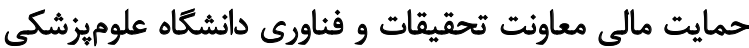

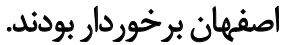

\section{مشاركت نويسيندكَان}

طراحى و ايدهيردازى مطالعه: جاويد مستمند، زهره

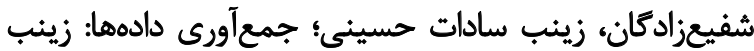

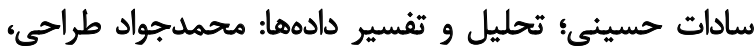

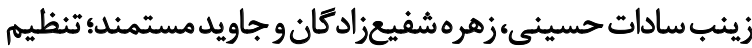

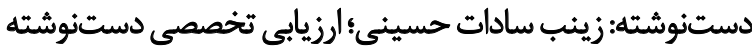

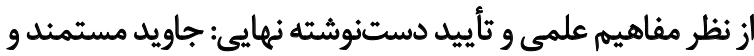

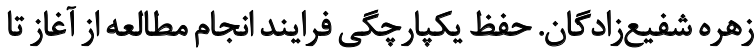
انتشار و ياسخ كويى به نظرات داوران: جاويد مستمند.
سيستم حسى و كنترل حركتي، نتايج مطالعات نيز نشاندهنده

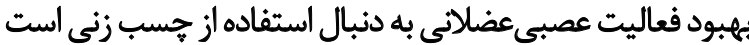

[rq. $4 q .98 .8 \mathrm{~V}]$

حس عمقى به عنوان يكى از اجزاي مهمم ثبات ديناميك مفصل

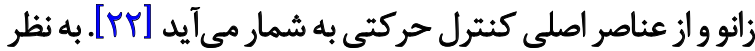

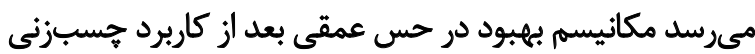

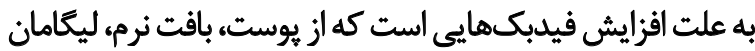

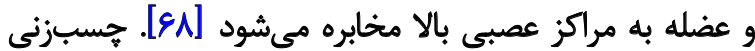

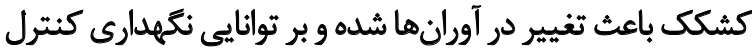

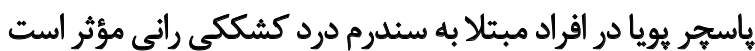

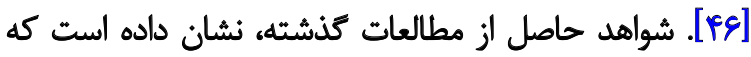

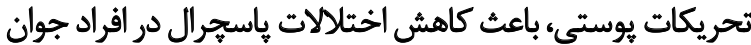

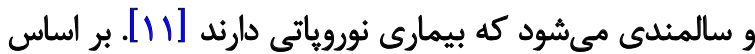

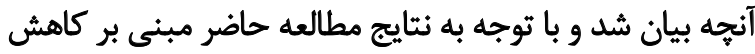

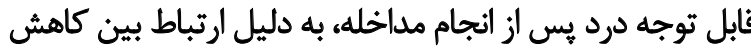

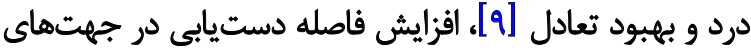

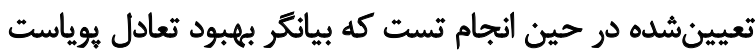

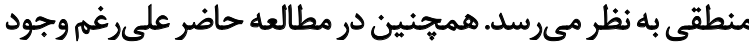

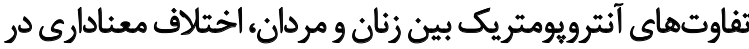

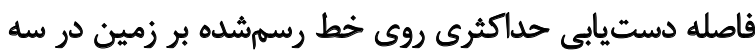

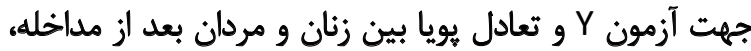

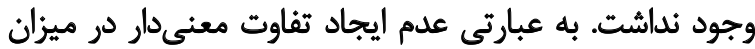

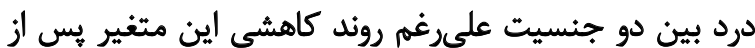

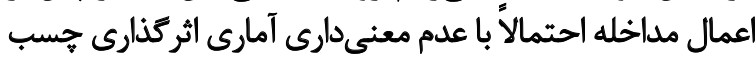

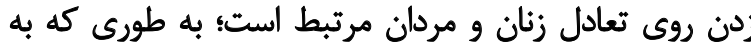

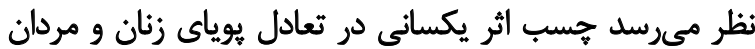

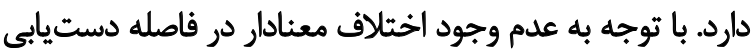

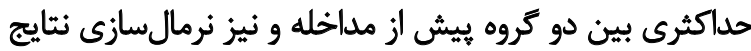

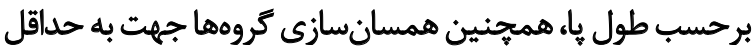

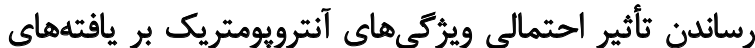

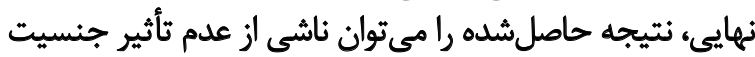
بر ميزان تأثيرات جسب كينزئه

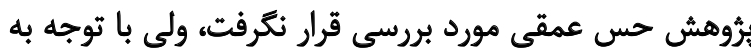

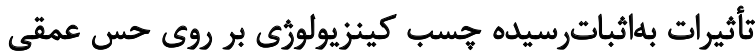

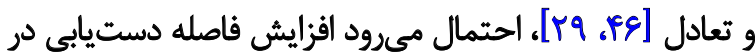

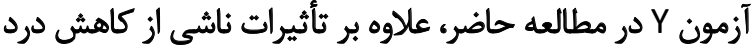

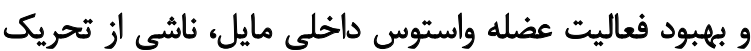

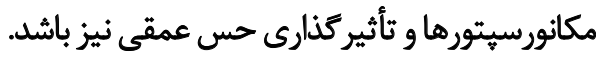

\section{تيجيجئيرى}

اكرجه يافتههاى برؤهش حاضر بيانكر يكسان بودن تأثير

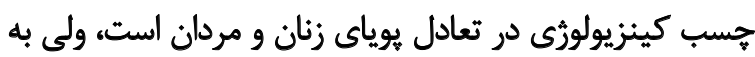

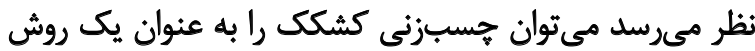




$$
\text { ثعارض مناقع }
$$

بنابر اظهار نويسندكان اين مقاله تعارض منافع ندارد.

$$
\text { تشكر و قدردانى }
$$

بدينوسيله از جناب آقاى دكتر خادم و كلينيك فيزيوترايى

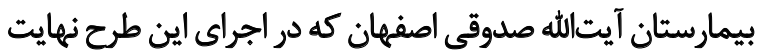

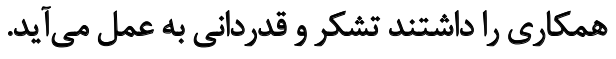


References

[1] Crossley K, Bennell K, Green S, Cowan S, McConnell J. Physical therapy for patellofemoral pain. The American Journal of Sports Medicine. 2002; 30(6):857-65. [DOI:10.1177/036354650203000 61701] [PMID]

[2] Petersen W, Ellermann A, Gösele-Koppenburg A, Best R, Rembitzki IV, Brüggemann GP, et al. Patellofemoral pain syndrome. Knee Surgery, Sports Traumatology, Arthroscopy. 2014; 22(10):2264-74. [DOI:10.1007/s00167-013-2759-6] [PMID] [PMCID]

[3] Barton C, Balachandar V, Lack S, Morrissey D. Patellar taping for patellofemoral pain: A systematic review and meta-analysis to evaluate clinical outcomes and biomechanical mechanisms. British Journal of Sports Medicine. 2013; 48(6):407. [DOI:10.1136/ bjsports-2013-092437] [PMID]

[4] Fagan V, Delahunt E. Patellofemoral pain syndrome-a review on the associated neuromuscular deficits and current treatment options. British Journal of Sports Medicine. 2008; 42(10):789-95. [DOI:10.1136/bjsm.2008.046623] [PMID]

[5] McConnell J. The management of chondromalacia patellae: A long term solution. Australian Journal of Physiotherapy. 1986; 32(4):215-23. [DOI:10.1016/S0004-9514(14)60654-1]

[6] Witrouw E, Lysens R, Bellemans J, Cambier D, Vandersteraeten $\mathrm{J}$. Intrinsic risk factors for the development of anterior knee pain in an athletic population. American Journal of Sports Medicine. 2000; 28(4):480-8. [DOI:10.1177/03635465000280040701 ] [PMID]

[7] Logan CA, Bhashyam AR, Tisosky AJ, Haber DB, Jorgensen A, Roy A, et al. Systematic review of the effect of taping techniques on patellofemoral pain syndrome. Sports Health. 2017; 9(5):45661. [DOI:10.1177/1941738117710938] [PMID] [PMCID]

[8] Aghapour E, Kamali F, Sinaei E. Effects of Kinesio Taping® on knee function and pain in athletes with patellofemoral pain syndrome. Journal of Bodywork and Movement Therapies. 2017; 21(4):835-9. [DOI:10.1016/j.jbmt.2017.01.012] [PMID]

[9] Lim EH, Kim ME, Kim SH, Park KN. Effects of posterior X taping on movement quality and knee pain intensity during forward-step-down in patients with patellofemoral pain syndrome. Journal of Sports Science and Medicine. 2020; 19(1):224. [PMCID] [PMID

[10] Mostamand J, Bader DL, Hudson Z. Reliability testing of the Patellofemoral Joint Reaction Force (PFJRF) measurement during double-legged squatting in healthy subjects: A pilot study. Journal of Bodywork and Movement Therapies. 2012; 16(2):21723. [DOI:10.1016/j.jbmt.2011.03.003] [PMID]

[11] Osorio JA, Vairo GL, Rozea GD, Bosha PJ, Millard RL, Aukerman DF, et al. The effects of two therapeutic patellofemoral taping techniques on strength, endurance, and pain responses. Physical Therapy in Sport. 2013; 14(4):199-206. [DOI:10.1016/j. ptsp.2012.09.006] [PMID]

[12] de Moura Campos Carvalho ESAP, Peixoto Leao Almeida G, Oliveira Magalhaes M, Renovato Franca FJ, Vidal Ramos LA, Comachio J, et al. Dynamic postural stability and muscle strength in patellofemoral pain: Is there a correlation? Knee. 2016; 23(4):61621. [DOI:10.1016/j.knee.2016.04.013] [PMID]
[13] Etemadi M, Asadi Z, Hedayati R, Salavati M, Aminian far A. Effects of the surface instability degrees on dynamic postural stability in anterior knee pain patients and healthy subjects. Koomesh Journal. 2013; 15(1):67-77. http://koomeshjournal.semums.ac.ir/ article-1-1457-en.html

[14] Levinger P, Gilleard W, Coleman C. Femoral medial deviation angle during a one-leg squat-test in individuals with patellofemoral pain syndrome. Physical Therapy in Sport. 2007; 8(4):163-8. DOI:10.1016/j.ptsp.2007.03.003

[15] Souza RB, Draper CE, Fredericson M, Powers CM. Femur rotation and patellofemoral joint kinematics: A weight-bearing magnetic resonance imaging analysis. Journal of Orthopaedic \& Sports Physical Therapy. 2010; 40(5):277-85. [DOI:10.2519/ jospt.2010.3215] [PMID]

[16] Motealleh A, Mohamadi M, Moghadam MB, Nejati N, Arjang $\mathrm{N}$, Ebrahimi N. Effects of core neuromuscular training on pain, balance, and functional performance in women with patellofemoral pain syndrome: A clinical trial. Journal of Chiropractic Medicine. 2019; 18(1):9-18. [DOI:10.1016/j.jcm.2018.07.006] [PMID] [PMCID]

[17] Reimer RC, Wikstrom EA. Functional fatigue of the hip and ankle musculature cause similar alterations in single leg stance postural control. Journal of Science and Medicine in Sport. 2010; 13(1):161-6. [DOI:10.1016/j.jsams.2009.01.001] [PMID]

[18] Salavati M, Moghadam M, Ebrahimi I, Arab AM. Changes in postural stability with fatigue of lower extremity frontal and sagittal plane movers. Gait Posture. 2007; 26(2):214-8. [DOI:10.1016/j. gaitpost.2006.09.001] [PMID]

[19] Mokhtari-Nia HR, Ebrahimi E, Salavati M. [Comparative criteria study of dynamic balancing in pateints with patello-femoral pain (Persian)]. Archives of Rehabilitation. 2005; 6(3):33-7. http://rehabilitationj.uswr.ac.ir/article-1-80-fa.html

[20] Hosseini SH, Anbarian M, Farahmand F, Ansari M. [Effect of kneeisokinetic extension training with maximum lateral tibial rotation on vastus amplitudes in patellofemoral pain syndrome patients (Persian)]. Archives of Rehabilitation. 2016; 16(4):312-23 http://rehabilitationj.uswr.ac.ir/article-1-1623-fa.html

[21] Akseki D, Akkaya G, Erduran M, Pinar H. Proprioception of the knee joint in patellofemoral pain syndrome. Acta Orthopaedica et Traumatologica Turcica. 2008; 42(5):316-21. [DOI:10.3944/ AOT'T.2008.316] [PMID]

[22] Rezazadeh F, E'zzati K, Karimi N, Vali-Zadeh A. [Comparison of the balance indices of professional athletes with and without Patellofemoral Pain Syndrome (Persian)]. Archives of Rehabilitation. 2012; 13(2):49-55. http://rehabilitationj.uswr.ac.ir/article1-1044-fa.html

[23] Salavati M. [Postural control abnormalities in patient with chronic low back pain: Effects of active specific spinal stabilization exercise (Persian)]. [PhD. Dissertation]. Tehran: Taebiat Modarres University; 2002.

[24] Kramer J, Handfield T, Kiefer G, Forwell L, Birmingham T. Comparisons of weight-bearing and non-weight-bearing tests of knee proprioception performed by patients with patello-femoral pain syndrome and asymptomatic individuals. Clinical Journal of Sport Medicine. 1997; 7(2):113-8. DOI:10.1097/00042752199704000-00007] [PMID] 
[25] Goto S. The effect of ptellofemoral pain syndrome on the hip and knee neuromuscular control on dynamic postural control task [PhD. dissertation]. Toledo: University of Toledo; 2009. http:// rave.ohiolink.edu/etdc/view?acc_ num=toledo1 254078175

[26] Negahban H, Etemadi M, Naghibi S, Emrani A, Yazdi MJS, Salehi R, et al. The effects of muscle fatigue on dynamic standing balance in people with and without patellofemoral pain syndrome. Gait Posture. 2013; 37(3):336-9. [DOI:10.1016/j.gaitpost.2012.07.025] [PMID]

[27] Aytar A, Ozunlu N, Surenkok O, Baltacı G, Oztop P, Karatas M. Initial effects of kinesio ${ }^{\circledR}$ taping in patients with patellofemoral pain syndrome: A randomized, double-blind study. Isokinetics and Exercise Science. 2011; 19(2):135-42. [DOI:10.3233/IES2011-0413]

[28] Kannus P, Natri A, Paakkala T, JÄrvinen M. An outcome study of chronic patellofemoral pain syndrome. Seven-year follow-up of patients in a randomized, controlled trial. Journal of Bone and Joint Surgery. 1999; 81(3):355-63. [DOI:10.2106/00004623199903000-00007] [PMID]

[29] Chang WD, Chen FC, Lee CL, Lin HY, Lai PT. Effects of Kinesio taping versus McConnell taping for patellofemoral pain syndrome: A systematic review and meta-analysis. EvidenceBased Complementary and Alternative Medicine. 2015; 2015. [DOI:10.1155/2015/471208] [PMID] [PMCID]

[30] Ebrahimi Atri A, Dehghani Tafti M, Khoshraftare Yazdi N, Dehghani Tafti V. [The effects of patellar taping on dynamic balance and reduction of pain in athletic women with Patellofemoral Pain Syndrome (PFPS) (Persiaan)]. The Journal of Shahid Sadoughi University of Medical Sciences. 2012; 20(3):332-39. https:/ /www. magiran.com/paper/1038741/?lang=en

[31] Mostamand J, Bader DL, Hudson Z. The effect of patellar taping on joint reaction forces during squatting in subjects with Patellofemoral Pain Syndrome (PFPS). Journal of Bodywork and Movement Therapies. 2010; 14(4):375-81. [DOI:10.1016/j. jbmt.2009.07.003] [PMID]

[32] Callaghan MJ, McKie S, Richardson P, Oldham JA. Effects of patellar taping on brain activity during knee joint proprioception tests using functional magnetic resonance imaging. Physical Therapy. 2012; 92(6):821-30. [DOI:10.2522/ptj.20110209] [PMID] [PMCID]

[33] Shafizadegan Z, Baharlouei H, Khoshavi O, Garmabi Z, Fereshtenejad N. Evaluating the short-term effects of Kinesiology taping and Stretching of Gastrocnemius on postural control: A randomized clinical trial. Journal of Bodywork and Movement Therapies. 2019; 24(2):196-201. [DOI:10.1016/j. jbmt.2019.11.003] [PMID]

[34] Jaraczewska E, Long C. Kinesio ${ }^{\circledR}$ taping in stroke: Improving functional use of the upper extremity in hemiplegia. Topics in Stroke Rehabilitation. 2006; 13(3):42-31. [DOI:10.1310/33KAXYE3-QWJB-WGT6] [PMID]

[35] Baharlouei H, Khoshavi O, Garmabi Z, Fereshtenejad N, Shafizadegan Z. Comparing the immediate effects of kinesiotaping and stretching of gastrocnemius on balance in elderly. Journal of Mazandaran University of Medical Sciences. 2017; 27(149):99-110. https://www.cochranelibrary.com/es/central/ doi/10.1002/central/CN-01733733/full
[36] Ebrahimi Takamjani E, Salavati M, Mokhtari Nia H, Dadgoo $\mathrm{M}$. The effect of patellar taping on knee joint proprioception in PFPS and healthy subjects. Razi Journal of Medical Sciences. 2004; 11(40):185-93. http://rjms.iums.ac.ir/browse.php?a_ id $=2 \&$ sid $=1 . \&$ slc_lang=en

[37] Tsai CT, Chang WD, Lee JP. Effects of short-term treatment with kinesiotaping for plantar fasciitis. Journal of Musculoskeletal Pain. 2010; 18(1):71-80. [DOI:10.3109/10582450903495882]

[38] Song CY, Lin JJ, Chang AH. Effects of femoral rotational taping on dynamic postural stability in female patients with patellofemoral Pain. Clinical Journal of Sport Medicine. 2017; 27(5):438-43. [DOI:10.1097/JSM.0000000000000392] [PMID]

[39] Miller J, Westrick R, Diebal A, Marks C, Gerber JP. Immediate effects of lumbopelvic manipulation and lateral gluteal kinesio taping on unilateral patellofemoral pain syndrome: A pilot study. Sports Health. 2013; 5(3):214-9 . [DOI:10.1177/1941738112473561] [PMID] [PMCID]

[40] Rozzi SL, Lephart SM, Gear WS, Fu FH. Knee joint laxity and neuromuscular characteristics of male and female soccer and basketball players. The American Journal of Sports Medicine. 1999; 27(3):312-9. [DOI:10.1177/03635465990270030801] [PMID]

[41] Chappell JD, Yu B, Kirkendall DT, Garrett WE. A comparison of knee kinetics between male and female recreational athletes in stop-jump tasks. The American journal of sports medicine. 2002; 30(2):261-7. [DOI:10.1177/03635465020300021901] [PMID]

[42] Prins MR, Van der Wurff P. Females with patellofemoral pain syndrome have weak hip muscles: A systematic review. Australian Journal Of Physiotherapy. 2009; 55(1):9-15. [DOI:10.1016/ S0004-9514(09)70055-8]

[43] Ford KR, Myer GD, Smith RL, Vianello RM, Seiwert SL, Hewett TE. A comparison of dynamic coronal plane excursion between matched male and female athletes when performing single leg landings. Clinical Biomechanics. 2006; 21(1):33-40. [DOI:10.1016/j.clinbiomech.2005.08.010] [PMID]

[44] Holden S, Boreham C, Doherty C, Wang D, Delahunt E. Dynamic postural stability in young adolescent male and female athletes. Pediatric Physical Therapy. 2014; 26(4):447-52. [DOI:10.1097/PEP.0000000000000071] [PMID]

[45] Mokhtarinia H, Ebrahimi-Takamjani I, Salavati M, Goharpay S, Khosravi A. The effect of patellar taping on knee joint proprioception in patients with patellofemoral pain syndrome. Acta Medica Iranica. 2008; 46(3):183-90. https://www.sid.ir/en/Jour$\mathrm{nal} /$ ViewPaper.aspx?ID=109611

[46] Aminaka N, Gribble PA. Patellar taping, patellofemoral pain syndrome, lower extremity kinematics, and dynamic postural control. Journal of athletic training. 2008; 43(1):21-8. [DOI:10.4085/10626050-43.1.21] [PMID] [PMCID]

[47] Citaker S, Kaya D, Yuksel I, Yosmaoglu B, Nyland J, Atay OA, et al. Static balance in patients with patellofemoral pain syndrome. Sports Health. 2011; 3(6):524-7. [DOI:10.1177/1941738111420803] [PMID] [PMCID]

[48] Baker V, Bennell K, Stillman B, Cowan S, Crossley K. Abnormal knee joint position sense in individuals with patellofemoral pain syndrome. Journal of Orthopaedic Research. 2002; 20(2):208-14. [DOI:10.1016/S0736-0266(01)00106-1] 
[49] Herrington L. The inter-tester reliability of a clinical measurement used to determine the medial/lateral orientation of the patella. Manual Therapy. 2002; 7(3):163-7. [DOI:10.1054/ math.2002.0463] [PMID]

[50] Toulotte C, Thevenon A, Fabre C. Effects of training and detraining on the static and dynamic balance in elderly fallers and non-fallers: A pilot study. Disability and Rehabilitation. 2006; 28(2):125-33. [DOI:10.1080/09638280500163653] [PMID]

[51] Messier SP, Glasser JL, Ettinger WH, Craven TE, Miller ME. Declines in strength and balance in older adults with chronic knee pain: A 30-month longitudinal, observational study. Arthritis Care \& Research (Hoboken). 2002; 47(2):141-8. [DOI:10.1002/ art.10339] [PMID]

[52] Hertel J, Miller SJ, Denegar CR. Intratester and intertester reliability during the Star Excursion Balance Tests. Journal of Sport Rehabilitation. 2000; 9(2):104-16. [DOI:10.1123/jsr.9.2.104]

[53] Robinson RH, Gribble PA. Support for a reduction in the number of trials needed for the star excursion balance test. Archives of Physical Medicine and Rehabilitation. 2008; 89(2):364-70. [DOI:10.1016/j.apmr.2007.08.139] [PMID]

[54] Gribble PA, Hertel J. Considerations for normalizing measures of the Star Excursion Balance Test. Measurement in Physical Education and Exercise Science. 2003; 7(2):89-100. [DOI:10.1207/ S15327841MPEE0702_3]

[55] Bouillon LE, Baker JL. Dynamic balance differences as measured by the star excursion balance test between adult-aged and middle-aged women. Sports Health. 2011; 3(5):466-9. [DOI:10.1177/1941738111414127] [PMID] [PMCID]

[56] Plisky PJ, Gorman PP, Butler RJ, Kiesel KB, Underwood FB, Elkins B. The reliability of an instrumented device for measuring components of the star excursion balance test. North American Journal Of Sports Physical Therapy: NAJSPT. 2009; 4(2):92-9. [PMCID] [PMID]

[57] Kahle NL, Gribble PA. Core stability training in dynamic balance testing among young, healthy adults. Athletic Training and Sports Health Care. 2009; 1(2):65-73. [DOI:10.3928/1942586420090301-03

[58] Kase K, Wallis J, Kenzo K. Clinical therapeutic applications of the Kinesio taping methods. Kinesio: Kinesio Taping Association International; 2013. https://books.google.com/books?id=TW_ cSAAACAAJ\&dq $=$

[59] Riemann BL, Lephart SM. The sensorimotor system, part I: The physiologic basis of functional joint stability. Journal of Athletic Training. 2002; 37(1):71-9. [PMCID] [PMID]

[60] Shumway-Cook A, Woollacott MH. Motor control: Translating research into clinical practice. Philadelphia: Lippincott Williams \& Wilkins; 2007. https://books.google.com/books?hl=en\&lr=\&id $=$ BJcL3enz3xMC\&oi $=$ fnd\&pg $=$ PA1\&dq $=$

[61] Hassan B, Mockett S, Doherty M. Static postural sway, proprioception, and maximal voluntary quadriceps contraction in patients with knee osteoarthritis and normal control subjects. Annals of the Rheumatic Diseases. 2001; 60(6):612-8. [DOI:10.1136/ ard.60.6.612] [PMID] [PMCID]
[62] Bennell K, Wee E, Crossley K, Stillman B, Hodges P. Effects of experimentally-induced anterior knee pain on knee joint position sense in healthy individuals. Journal of Orthopaedic Research. 2005; 23(1):46-53. [DOI:10.1016/j.orthres.2004.06.008] [PMID]

[63] Ernst GP, Kawaguchi J, Saliba E. Effect of patellar taping on knee kinetics of patients with patellofemoral pain syndrome. Journal of Orthopaedic \& Sports Physical Therapy. 1999; 29(11):661-7. [DOI:10.2519/jospt.1999.29.11.661] [PMID]

[64] Campolo M, Babu J, Dmochowska K, Scariah S, Varughese J. A comparison of two taping techniques (kinesio and mcconnell) and their effect on anterior knee pain during functional activities. International Journal of Sports Physical Therapy. 2013; 8(2):10510. https://pubmed.ncbi.nlm.nih.gov/23593548/

[65] Earl J, Hertel J, Denegar C. Efficacy of a 6-week neuromuscular rehabilitation program on pain, function, muscle activity and joint motion in patients with patellofemoral pain. Journal of Athletic Training. 2002; 38(suppl 2):83. https://www.sid.ir/en/journal/ ViewPaper.aspx?ID $=277531$

[66] Powers CM, Landel R, Sosnick T, Kirby J, Mengel K, Cheney A, et al. The effects of patellar taping on stride characteristics and joint motion in subjects with patellofemoral pain. Journal of Orthopaedic \& Sports Physical Therapy. 1997; 26(6):286-91. [DOI:10.2519/jospt.1997.26.6.286] [PMID]

[67] Yosmaoglu HB, Kaya D, Guney H, Nyland J, Baltaci G, Yuksel I, et al. Is there a relationship between tracking ability, joint position sense, and functional level in patellofemoral pain syndrome? Knee Surg Sports Traumatol Arthrosc. 2013; 21(11):2564-71. [DOI:10.1007/s00167-013-2406-2] [PMID]

[68] Cho HY, Kim EH, Kim J, Yoon YW. Kinesio taping improves pain, range of motion, and proprioception in older patients with knee osteoarthritis: A randomized controlled trial. American Journal of Physical Medicine \& Rehabilitation. 2015; 94(3):192-200. [DOI:10.1097/PHM.0000000000000148] [PMID] 
This Page Intentionally Left Blank 